\title{
Spatial Topography of Individual-Specific Cortical Networks Predicts Human Cognition, Personality, and Emotion
}

\author{
Ru Kong ${ }^{1}$, Jingwei Li ${ }^{1}$, Csaba Orban ${ }^{1}$, Mert R. Sabuncu ${ }^{2}$, Hesheng Liu ${ }^{8}$, \\ Alexander Schaefer ${ }^{1}$, Nanbo Sun ${ }^{1}$, Xi-Nian Zuo ${ }^{3,4}$, Avram J. Holmes ${ }^{5}$, \\ Simon B. Eickhoff6,7 and B.T. Thomas Yeo (D) 1,8,9,10
}

${ }^{1}$ Department of Electrical and Computer Engineering, ASTAR-NUS Clinical Imaging Research Centre, Singapore Institute for Neurotechnology and Memory Networks Program, National University of Singapore, Singapore, ${ }^{2}$ School of Electrical and Computer Engineering, Cornell University, Ithaca, NY, USA, ${ }^{3} \mathrm{CAS}$ Key Laboratory of Behavioral Sciences and Research Center for Lifespan Development of Brain and Mind (CLIMB), Institute of Psychology, Beijing, China, ${ }^{4}$ University of Chinese Academy of Sciences, Beijing, China, ${ }^{5}$ Department of Psychology, Yale University, New Haven, CT, USA, ${ }^{6}$ Institute for Systems Neuroscience, Medical Faculty, Heinrich-Heine University Düsseldorf, Düsseldorf, Germany, ${ }^{7}$ Institute of Neuroscience and Medicine, Brain \& Behaviour (INM-7), Research Center Jülich, Jülich, Germany, ${ }^{8}$ Martinos Center for Biomedical Imaging, Massachusetts General Hospital, Charlestown, MA, USA, ${ }^{9}$ Centre for Cognitive Neuroscience, DukeNUS Medical School, Singapore and ${ }^{10}$ NUS Graduate School for Integrative Sciences and Engineering, National University of Singapore, Singapore

Address correspondence to: B.T. Thomas Yeo, ECE, ASTAR-NUS CIRC, SINAPSE \& MNP, National University of Singapore, Singapore. Email: thomas.yeo@nus.edu.sg (1D orcid.org/0000-0002-0119-3276

\begin{abstract}
Resting-state functional magnetic resonance imaging (rs-fMRI) offers the opportunity to delineate individual-specific brain networks. A major question is whether individual-specific network topography (i.e., location and spatial arrangement) is behaviorally relevant. Here, we propose a multi-session hierarchical Bayesian model (MS-HBM) for estimating individualspecific cortical networks and investigate whether individual-specific network topography can predict human behavior. The multiple layers of the MS-HBM explicitly differentiate intra-subject (within-subject) from inter-subject (between-subject) network variability. By ignoring intra-subject variability, previous network mappings might confuse intra-subject variability for inter-subject differences. Compared with other approaches, MS-HBM parcellations generalized better to new rs-fMRI and task-fMRI data from the same subjects. More specifically, MS-HBM parcellations estimated from a single rs-fMRI session (10 $\mathrm{min}$ ) showed comparable generalizability as parcellations estimated by 2 state-of-the-art methods using 5 sessions (50 min). We also showed that behavioral phenotypes across cognition, personality, and emotion could be predicted by individual-specific network topography with modest accuracy, comparable to previous reports predicting phenotypes based on connectivity strength. Network topography estimated by MS-HBM was more effective for behavioral prediction than network size, as well as network topography estimated by other parcellation approaches. Thus, similar to connectivity strength, individual-specific network topography might also serve as a fingerprint of human behavior.
\end{abstract}


Key words: behavior prediction, brain parcellation, individual differences, network topography, resting-state functional connectivity

\section{Introduction}

One prominent tool for identifying large-scale human brain networks is resting-state functional connectivity (RSFC), which reflects the synchrony of fMRI signals between brain regions, while a subject is lying at rest without any goal-directed task (Biswal et al. 1995; Greicius et al. 2003; Fox and Raichle 2007). RSFC brain networks correspond well to task-evoked activation patterns (Seeley et al. 2007; Smith et al. 2009; Cole et al. 2014; Yeo, Krienen, et al. 2015a). RSFC is also heritable (Glahn et al. 2010; Yang et al. 2016; Ge et al. 2017), correlates with gene expression across the cortical mantle (Hawrylycz et al. 2015; Richiardi et al. 2015; Krienen et al. 2016), and predicts individual differences in behavior (Hampson et al. 2006; van den Heuvel et al. 2009; Finn et al. 2015; Smith et al. 2015). Consequently, RSFC has been widely utilized to estimate population-average functional brain networks by averaging data across multiple subjects (Beckmann et al. 2005; Damoiseaux et al. 2006; Fox et al. 2006; Dosenbach et al. 2007; Margulies et al. 2007; Power et al. 2011; Yeo et al. 2011; Lee et al. 2012).

Population-average networks have provided important insights into the large-scale functional organization of the human brain (Buckner et al. 2013; Wig 2017). However, since population-average networks might obscure individual-specific network organization, there is significant interest in estimating individual-specific brain networks (Beckmann et al. 2009; Bellec et al. 2010; Zuo et al. 2010; Varoquaux et al. 2011; Hacker et al. 2013; Wig et al. 2014; Chong et al. 2017). Indeed, many studies have documented that the size, location, and spatial arrangement of individual-specific brain networks vary substantially across participants (Harrison et al. 2015; Laumann et al. 2015; Wang et al. 2015; Glasser et al. 2016; Braga and Buckner 2017; Gordon, Laumann, Adeyemo, Petersen, et al. 2017a; Gordon, Laumann, Adeyemo, Gilmore, et al. 2017b; Gordon, Laumann, Gilmore, Newbold, et al. 2017c). Yet, the possible behavioral relevance of individual differences in network size and network topography (location and spatial arrangement) remains largely unclear.

We proposed a multi-session hierarchical Bayesian model (MS-HBM) for estimating individual-specific network parcellations of the cerebral cortex and investigated whether individual-specific network topography and size were associated with human behavior. The multiple layers of the MS-HBM allowed explicit separation of inter-subject (between-subject) and intra-subject (within-session) functional connectivity variability. Previous individual-specific network mappings only accounted for inter-subject variability, but not intra-subject variability. However, inter-subject and intra-subject RSFC variability can be markedly different across regions (Mueller et al. 2013; Chen et al. 2015; Laumann et al. 2015). For example, the motor cortex exhibits high intra-subject functional connectivity variability, but low inter-subject functional connectivity variability (Laumann et al. 2015). Therefore, observed RSFC variability in the motor cortex might be incorrectly attributed to inter-subject variability of brain networks, rather than just intra-subject sampling variability, resulting in sub-optimal network mapping. In this paper, we showed that compared with 4 other approaches, MS-HBM individual-specific parcellations generalized better to new resting and task-fMRI data from the same individuals.

Having established that the MS-HBM generated high-quality individual-specific parcellations, we investigated whether individual differences in network topography (i.e., location and spatial arrangement) and size could predict behavioral measures across cognition, personality and emotion. While there is a plethora of studies associating regional brain volumes and anatomical patterns with behavior (e.g., Erickson et al. 2011; Holmes et al. 2016; Sabuncu et al. 2016; Cachia et al. 2017), there are relatively few studies relating topography and size of functional areas (or networks) with behavior or other traits (Dehaene et al. 2010; Bijsterbosch et al. 2018; Salehi et al. 2018). Using kernel regression, we showed that multiple behavioral measures could be predicted with modest accuracy. Furthermore, network topography estimated by MS-HBM achieved better prediction accuracies than topography estimated by other parcellation approaches. Lastly, we found that at least at the resolution of large-scale networks, network topography was more useful than network size in predicting behavior.

The contributions of this work are 2-fold. First, by estimating inter-subject variability, intra-subject variability and individualspecific networks within a unified statistical framework, the estimation of individual-specific networks was greatly improved. For example, MS-HBM parcellations estimated from a single rs-fMRI session were comparable to those generated by 2 prominent algorithms using 5 times the amount of data (Wang et al. 2015; Gordon, Laumann, Adeyemo, Petersen, et al. 2017a; Gordon, Laumann, Adeyemo, Gilmore, et al. 2017b), as evaluated by generalizability to new rs-fMRI data from the same subjects. Second, our results suggest that individual-specific cortical network topography might serve as a fingerprint of human behavior, which might complement the usage of functional connectivity strength in the vast majority of previous literature (Hampson et al. 2006; Finn et al. 2015; Rosenberg et al. 2016; Smith et al. 2015; Yeo, Tandi, et al. 2015b, Nostro et al. 2018). This highlights the importance of considering both network topography and functional connectivity strength for behavioral prediction.

\section{Methods}

\section{Overview}

We proposed a MS-HBM to estimate functional network parcellations of the cerebral cortex in individual subjects. The model distinguished between inter-subject and intra-subject network variabilities. Subsequent analyses proceeded in 4 stages. First, to examine whether inter-subject and intra-subject variabilities could be reliably estimated across datasets, the MS-HBM was applied to 3 multi-session rs-fMRI datasets. Second, the MSHBM was compared with 4 other approaches using new rs-fMRI and task-fMRI data from the same subjects. Third, we examined the reproducibility of the MS-HBM parcellations and how well the parcellations captured inter-subject differences. Finally, we investigated whether individual differences in cortical parcellations reflected individual differences in behavior.

\section{Multi-session rs-fMRI Datasets}

The Genomic Superstruct Project (GSP) test-retest dataset (Holmes et al. 2015) consisted of structural MRI and rs-fMRI from 69 healthy young adults (ages 18-35). All imaging data were collected on matched $3 \mathrm{~T}$ Tim Trio scanners (Siemens 
Healthcare, Erlangen, Germany) at Harvard University and Massachusetts General Hospital using the vendor-supplied 12channel phased-array head coil. Each participant has 2 sessions, acquired on 2 different days separated by less than 6 months. One or 2 rs-fMRI runs were acquired per session. Each BOLD run was acquired in $3 \mathrm{~mm}$ isotropic resolution with a TR of $3.0 \mathrm{~s}$ and lasted for $6 \mathrm{~min}$ and $12 \mathrm{~s}$. The structural data consisted of one $1.2 \mathrm{~mm}$ isotropic scan for each session. Details of the data collection can be found elsewhere (Holmes et al. 2015).

The Hangzhou Normal University of the Consortium for Reliability and Reproducibility (CoRR-HNU) multi-session dataset (Zuo et al. 2014; Chen et al. 2015) consisted of structural MRI and rs-fMRI from 30 young healthy adults (ages 20-30). All imaging data were collected on a 3 T GE Discovery MR750 scanner using an 8-channel head coil. Each participant was scanned for a total of 10 sessions across 1 month (1 session every 3 days). One rs-fMRI run was collected in each session. Each fMRI run was acquired in $3.4 \mathrm{~mm}$ isotropic resolution with a TR of $2.0 \mathrm{~s}$ and lasted for $10 \mathrm{~min}$. The structural data consisted of one $1 \mathrm{~mm}$ isotropic scan for each session. Details of the data collection can be found elsewhere (Zuo et al. 2014; Chen et al. 2015).

The Human Connectome Project (HCP) S900 release (Van Essen, Ugurbil, et al. 2012b; Smith et al. 2013) consisted of structural MRI, rs-fMRI and task-fMRI of 881 subjects. All imaging data were collected on a custom-made Siemens $3 \mathrm{~T}$ Skyra scanner using a multiband sequence. Each participant went through 2 fMRI sessions on 2 consecutive days. Two rs-fMRI runs were collected in each session. Each fMRI run was acquired in $2 \mathrm{~mm}$ isotropic resolution with a TR of $0.72 \mathrm{~s}$ and lasted for $14 \mathrm{~min}$ and $33 \mathrm{~s}$. The structural data consisted of one $0.7 \mathrm{~mm}$ isotropic scan for each subject. Details of the data collection can be found elsewhere (Van Essen, Ugurbil, et al. 2012b; Smith et al. 2013).

It is worth noting the significant acquisition differences among the 3 datasets, including scanner type (e.g., GE vs. Siemens) and acquisition sequence (e.g., multiband vs. non-multiband). The interval between repeated visits was also very different, ranging from 1 day in the HCP dataset and up to 6 months in the GSP dataset. These differences allowed us to test the robustness of our individual-specific network estimation model.

\section{Preprocessing}

Processing of GSP and CoRR-HNU data followed the surfacebased pipeline of Yeo and colleagues (Yeo et al. 2011; Holmes et al. 2015) using a combination of FreeSurfer (Dale et al. 1999; Fischl, Sereno, Dale, et al. 1999a; Fischl, Sereno, Tootell, et al. 1999b; Fischl et al. 2001; Ségonne et al. 2007; Greve and Fischl 2009) and FSL (Jenkinson et al. 2002; Smith et al. 2004), with additional censoring steps pioneered by the Petersen's group (Power et al. 2014; Gordon et al. 2016). The final preprocessed data were on the FreeSurfer fsaverage 5 surface space $(4 \mathrm{~mm}$ vertex spacing). More details can be found in Supplementary Methods S1.

In the case of the HCP data, we utilized the MSMAll ICA-FIX data on fs_LR32K surface space (HCP S900 manual; Van Essen, Glasser, et al. 2012a; Van Essen, Ugurbil, et al. 2012b; Glasser et al. 2013; Smith et al. 2013; Griffanti et al. 2014; Salimi-Khorshidi et al. 2014) with additional nuisance regression, censoring (Burgess et al. 2016; Siegel et al., 2017) and spatial smoothing. More details can be found in Supplementary Methods S2.

\section{Population-Level Parcellation and Functional Connectivity Profiles}

We have previously developed an approach to derive a population-level parcellation of the cerebral cortex into large- scale resting-state networks (Yeo et al. 2011). The term "parcellation" is used to indicate that every cortical location is assigned a label (Fischl et al. 2004; Yeo et al. 2011), rather than delineating the locations of a specific brain function or network. The cortical networks were defined as sets of cortical regions with similar corticocortical functional connectivity profiles. Here we applied the same approach to the GSP, CoRRHNU and HCP datasets. Our previous analyses (Yeo et al. 2011) identified 7 and 17 networks to be particularly stable. For simplicity, we will only consider 17 networks throughout this paper. Details of this approach have been previously described (Yeo et al. 2011). For completeness, we will briefly describe its application to the current datasets.

Recall that the preprocessed fMRI data from the CoRR-HNU and GSP subjects have been projected onto the fsaverage5 surface meshes. The fsaverage 5 surface meshes consisted of 18715 cortical vertices. Following previous work (Yeo et al. 2011), the connectivity profile of a cortical region (vertex) was defined to be its functional coupling to 1175 regions of interest (ROIs). The 1175 ROIs consisted of single vertices uniformly distributed across the fsaverage 5 surface meshes. For each rs-fMRI run of each subject, the Pearson's correlation between the fMRI time series at each spatial location (18715 vertices) and the 1175 ROIs were computed. The $18715 \times 1175$ correlation matrix was then binarized by keeping the top $10 \%$ of the correlations to obtain the final functional connectivity profiles. Outlier volumes (flagged during preprocessing) were ignored when computing the correlations.

In the case of the HCP dataset, the preprocessed fMRI data have been projected onto the fs_LR surface space. The fs_LR32K surface meshes consisted of 59412 cortical vertices. We defined the connectivity profile of a cortical region (vertex) to be its functional coupling to 1483 ROIs. The 1483 ROIs consisted of single vertices uniformly distributed across the fs_LR32K surface meshes. For each rs-fMRI run of each subject, the Pearson's correlation between the fMRI time series at each spatial location (59 412 vertices) and the 1483 ROIs were computed. The $59412 \times 1483$ correlation matrix was then binarized by keeping the top $10 \%$ of the correlations to obtain the final functional connectivity profile. Outlier volumes were again ignored when computing the correlations.

To obtain a population-level parcellation from a group of subjects, each vertex's connectivity profiles were averaged across all BOLD runs of all subjects. The averaged connectivity profiles were then clustered using a mixture of von MisesFisher distributions (Lashkari et al. 2010; Yeo et al. 2011).

\section{Multi-session Hierarchical Bayesian Model}

The previous section described an approach to estimate a population-level parcellation from a group of subjects. Figure 1 illustrates the MS-HBM model for estimating individual-specific cerebral cortex parcellations using multi-session fMRI data. Let $\mathrm{X}_{n}^{\text {s,t }}$ denote the (binarized) functional connectivity profile of cortical vertex $n$ from session $t$ of subject $s$. For example, Figure 1 (fourth row) illustrates the binarized functional connectivity profile for a posterior cingulate cortex vertex $\left(X_{P C C}^{1,1}\right)$ and a precuneus vertex $\left(X_{p \text { Cun }}^{1,1}\right)$ from the first session of the first subject. The shaded circle indicates that $X_{n}^{s, t}$ is the only observation in the entire model. Based on the observed connectivity profiles of all vertices from all sessions of a single subject, the goal is to assign a network label $l_{n}^{s}$ for each vertex $n$ of subject s. Even though a vertex's connectivity profile is unlikely to be the same across different fMRI sessions, the vertex's network label was assumed to be the same across sessions. For example, Figure 1 


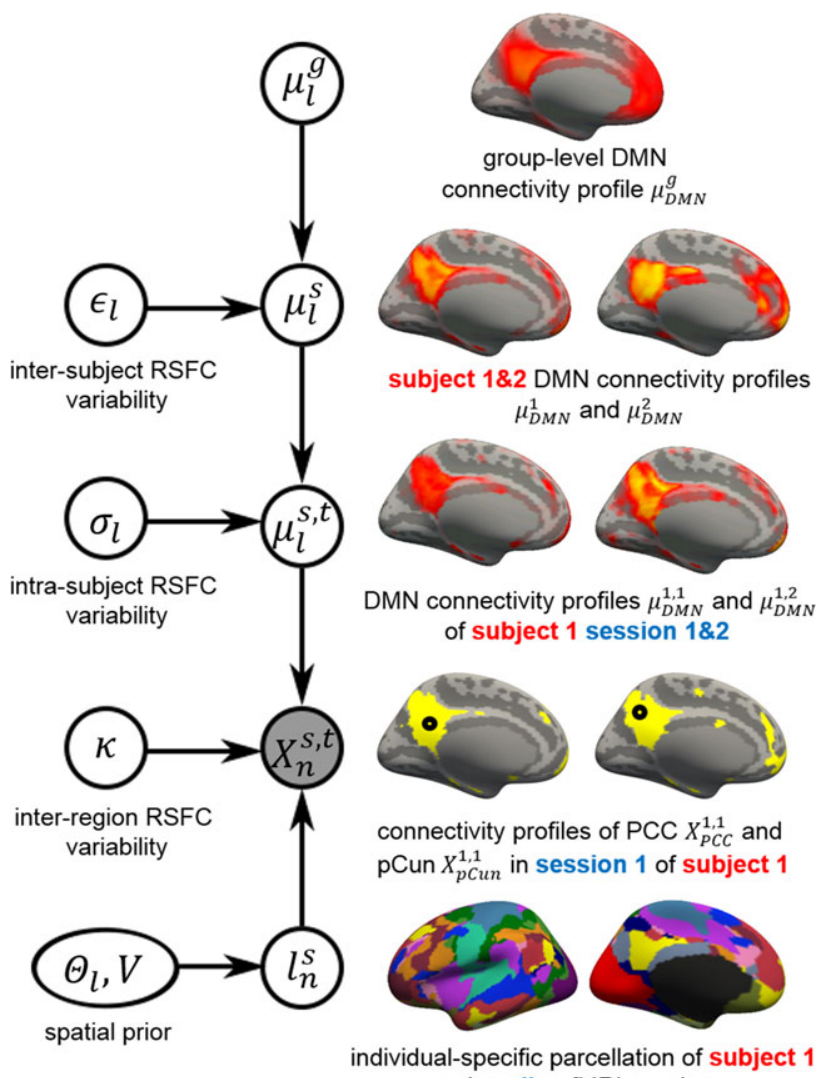

individual-specific parcellation of subject 1 using all rs-fMRI sessions

Figure 1. MS-HBM of individual-specific cortical parcellation. $X_{n}^{\text {s,t }}$ denote the RSFC profile at brain location $n$ of subject $s$ during rs-fMRI session $t$. The shaded circle indicates that $X_{n}^{s, t}$ are the only observed variables in the entire model. The goal is to estimate the network label $l_{n}^{s}$ for subject $s$ at each cortical location $n$ given RSFC profiles from all sessions. $\mu_{l}^{g}$ is the group-level RSFC profile of network l. $\mu_{l}^{\mathrm{S}}$ is the subject-specific RSFC profile of network l. A large $\epsilon$ indicates small inter-subject RSFC variability, i.e., the group-level and subject-specific RSFC profiles are very similar. $\mu_{l}^{\text {s,t }}$ is the subject-specific RSFC profile of network $l$ during session t. A large $\sigma_{l}$ indicates small intra-subject RSFC variability, i.e., the subject-level and session-level RSFC profiles are very similar. $\kappa$ captures inter-region RSFC variability. A large $\kappa$ indicates small inter-region variability, i.e., 2 regions from the same network exhibit very similar RSFC profiles. Finally, $\Theta_{l}$ captures inter-subject variability in the spatial distribution of networks (e.g., high probability of the DMN being located at the PCC), while smoothness prior $\mathrm{V}$ encourages network labels to be spatially smooth. See text for details.

(last row) illustrates the individual-specific parcellation of the first subject using data from all sessions.

Some of the model parameters (e.g., inter-subject variability) must be estimated from a training set of subjects. A new subject (possibly from another dataset) could then be parcellated without access to the original training data. Even though the model was defined on multi-session fMRI data, an effective workaround (details below) was provided for single-session fMRI data. The exact mathematical model is found in Supplemental Methods S3. Here we provide the intuition behind this model.

To obtain the subject-specific parcellation, the MS-HBM assumes that each cortical network exhibits a distinctive RSFC profile. Let $\mu_{l}^{g}$ denote the group-level functional connectivity profile of network l. We can think of $\mu_{1}^{g}$ as the average connectivity profile of all vertices of all sessions of all subjects belonging to network l. For example, Figure 1 (top row) illustrates the group-level default mode network (DMN) connectivity profile $\left(\mu_{\text {DMN }}^{g}\right)$.
To model inter-subject RSFC variability, let $\mu_{l}^{\mathrm{s}}$ denote the functional connectivity profile of network $l$ and subject $s$. We can think of $\mu_{1}^{\mathrm{s}}$ as the average connectivity profile of all vertices of all sessions of subject $s$ belonging to network l. For example, Figure 1 (second row) illustrates the DMN connectivity profiles of 2 subjects $\left(\mu_{\mathrm{DMN}}^{1}\right.$ and $\left.\mu_{\mathrm{DMN}}^{2}\right)$. The subject-specific connectivity profile $\mu_{l}^{\text {s }}$ was assumed to follow a von Mises-Fisher distribution with mean direction $\mu_{l}^{g}$ (group-level RSFC profile of network l) and concentration parameter $\epsilon_{l}$. A large $\epsilon_{l}$ indicates low inter-subject functional connectivity variability, i.e., $\mu_{1}^{\mathrm{s}}$ and $\mu_{1}^{g}$ are very similar. The subscript $l$ indicates that $\epsilon_{l}$ is different for each network.

To model intra-subject RSFC variability, let $\mu_{l}^{\text {s,t }}$ denote the functional connectivity profile of network $l$ and subject $s$ during session $t$. We can think of $\mu_{l}^{\text {s,t }}$ as the average connectivity profile of all vertices from session $t$ of subject $s$ belong to network l. For example, Figure 1 (third row) illustrates the DMN connectivity profiles of subject 1 during sessions 1 and $2\left(\mu_{\mathrm{DMN}}^{1,1}\right.$ and $\left.\mu_{\mathrm{DMN}}^{1,2}\right)$. The session-specific connectivity profiles $\mu_{l}^{\mathrm{s}, \mathrm{t}}$ was assumed to follow a von Mises-Fisher distribution with mean direction $\mu_{l}^{s}$ (subject-specific RSFC profile) and concentration parameter $\sigma_{l}$. A large $\sigma_{l}$ indicates low intra-subject functional connectivity variability, i.e., $\mu_{1}^{\mathrm{s}}$ and $\mu_{1}^{\mathrm{s}, \mathrm{t}}$ profiles are very similar. The subscript $l$ indicates that $\sigma_{l}$ is different for each network.

The observed connectivity profiles of 2 regions belonging to the same network are unlikely to be identical. For example, the connectivity profiles of PCC and precuneus are similar, but not identical (Fig. 1 fourth row) even though they might both belong to the DMN. To account for this intranetwork (interregion) variability, the observed connectivity profile $X_{n}^{s, t}$ of vertex $n$ (which has been assigned to network l) was assumed to follow a von Mises-Fisher distribution with mean direction $\mu_{1}^{\mathrm{s}, t}$ (session-specific and subject-specific connectivity profile of network l) and concentration parameter $\kappa$. A large $\kappa$ indicates low inter-region functional connectivity variability. Here, $\kappa$ was set to be the same across networks (see Supplementary Methods S3 for justification).

Given the previous modeling assumptions, if the observed connectivity profile $X_{n}^{s, t}$ of vertex $n$ was most similar to the session-specific and subject-specific connectivity profile $\mu_{l}^{\mathrm{s}, t}$ of the DMN (where similarity is measured via the likelihood of the von Mises-Fisher distribution), then vertex $n$ would be assigned to the DMN. Therefore, at this point, the model is somewhat similar to the population-level parcellation approach (Yeo2011), except that the population-level approach does not account for intra-subject and inter-subject RSFC variability. Furthermore, unlike group averaged connectivity profiles, the observed functional connectivity profiles of individual subjects are generally very noisy. If the observed profiles of PCC and pCun were too noisy, the model might not assign both of them to the DMN. Therefore, additional priors were imposed on the parcellation. First, the spatial smoothness prior $\mathrm{V}$ encourages neighboring vertices (e.g., PCC and pCun) to be assigned to the same network. Second, the spatial prior $\Theta_{l, n}$ denotes the probability of network $l$ occurring at a particular spatial location $n$. For example, PCC might have high prior probability of being assigned to the DMN.

Given a dataset of subjects with multi-session rs-fMRI data, the group-level network connectivity profiles $\mu_{l}^{9}$, the inter-subject functional connectivity variability $\epsilon_{l}$, the intra-subject functional connectivity variability $\sigma_{l}$, the spatial smoothness prior $\mathrm{V}$, and the inter-subject spatial variability prior $\Theta_{l}$ could be estimated. The estimated group-level priors $\left(\mu_{l}^{g}, \epsilon_{l}, \sigma_{l}, V, \Theta_{l}\right)$ could then be used to parcellate a new subject. Here we utilized a variational 
Bayes expectation-maximization (VBEM) algorithm to learn the group-level priors from the training data and to estimate individual-specific parcellations. Details of the VBEM algorithm can be found in Supplementary Methods S4.

Although the MS-HBM was formulated for multi-session fMRI data, most studies only collect a single run of fMRI data. We considered the ad-hoc approach of splitting the single fMRI run into 2 and treating the resulting runs as 2 separate sessions. Our evaluations (see Results) suggest that this workaround worked surprisingly well.

\section{Characterizing Inter-subject and Intra-subject Network Variability}

We first evaluate whether the MS-HBM can yield robust estimates of inter-subject and intra-subject variability across datasets. For the purpose of subsequent experiments, the GSP dataset was divided into training $(N=37)$ and validation $(N=$ 32) sets. The CoRR-HNU dataset $(N=30)$ was kept unchanged. The HCP dataset was divided into training $(N=40)$, validation $(N=40)$, and test $(N=596)$ sets. Furthermore, different fMRI runs within the same session were treated as data from different sessions. For example, each HCP subject underwent 2 fMRI sessions on 2 consecutive days. Within each session, there were 2 rs-fMRI runs. For the purpose of our analyses, we treated each HCP subject as having 4 sessions of data. Future work might differentiate between intrasession and intersession variability.

The group-level parcellation algorithm was applied to the GSP training set. The resulting group-level parcellation was then used to initialize the estimation of the group-level network connectivity profiles $\mu_{1}^{g}$, the inter-subject functional connectivity variability $\epsilon_{l}$, the intra-subject functional connectivity variability $\sigma_{l}$, and the inter-subject spatial variability prior $\Theta_{l}$. For the purpose of computational efficiency, the spatial smoothness prior $\mathrm{V}$ was ignored in this analysis (see Supplementary Methods S4.1 for justification). The procedure was repeated for the CoRR-HNU dataset and HCP training set.

\section{Comparison With Alternative Approaches}

Having confirmed previous literature (Mueller et al. 2013; Laumann et al. 2015) that inter-subject and intra-subject functional connectivity variability were indeed different across cortical networks, we compared MS-HBM with 4 alternative approaches. The first approach was to apply the populationlevel parcellation (Yeo et al. 2011) to individual subjects, which we will refer to as "Yeo2011". The second approach is "YeoBackProject", which is analogous to the ICA backprojection algorithm (Beckmann et al. 2009; Calhoun et al. 2009; Filippini et al. 2009; Zuo et al. 2010; Calhoun and Adali 2012). The third approach is the influential individual-specific parcellation algorithm of Gordon and colleagues (Gordon, Laumann, Adeyemo, Petersen, et al. 2017a; Gordon, Laumann, Adeyemo, Gilmore, et al. 2017b), which we will refer to as "Gordon2017". The fourth approach is the prominent individual-specific parcellation algorithm of Wang and colleagues (Wang et al. 2015), which we will refer to as "Wang2015". See Supplementary Methods S5 for more details.

All algorithms were applied to the CoRR-HNU dataset and the HCP test set. In the case of the CoRR-HNU dataset, the model parameters of all algorithms were estimated from the GSP dataset and then utilized to infer the parcellations of
CoRR-HNU subjects. This is important because inter-subject and intra-subject variabilities might differ across datasets, so it was important to evaluate whether MS-HBM model parameters estimated from one dataset could be generalized to another dataset. More specifically, the training procedure for the MSHBM was the same as the previous section, except that the GSP validation set was also used to tune the spatial smoothness prior V. Similarly, "free" parameters in Wang2015 and Gordon2017 were tuned using the GSP validation set.

In the case of the HCP dataset, recall that the HCP data were in a different surface space from the GSP data, so the GSP model parameters could not be applied to the HCP subjects. Instead, the model parameters of all algorithms were estimated from the HCP training and validation sets, and then utilized to infer the parcellation of each subject in the HCP test set.

\section{Quantitative Evaluation Measures}

Evaluating the quality of individual-specific resting-state parcellations is difficult because of a lack of ground truth. Here, we considered 2 common evaluation metrics utilized in previous studies (Gordon et al. 2016; Chong et al. 2017; Gordon, Laumann, Gilmore, Newbold, et al. 2017c; Schaefer et al. 2017): resting-state connectional homogeneity and task functional inhomogeneity measures. These metrics encode the principle that if an individual-specific parcellation captured the systemlevel organization of the individual's cerebral cortex, then each network should have homogeneous connectivity and function:

- Resting-state connectional homogeneity. Resting-state connectional homogeneity was computed by averaging the Pearson's correlations between the rs-fMRI time courses of all pairs of vertices within each network (Schaefer et al. 2017). The average correlations were then averaged across all networks while accounting for network size:

$$
\frac{\sum_{l=1}^{L} \rho_{l}|l|}{\sum_{l=1}^{L}|l|},
$$

where $\rho_{l}$ is the resting-state homogeneity of network $l$ and III is the number of vertices within network l (Schaefer et al. 2017). For each subject from CoRR-HNU $(N=30)$ and HCP test set $(N=596)$, we used one session to infer the individual-specific parcellation and computed the resting-state homogeneity of the individual-specific parcellation with the remaining sessions. Because the HNU dataset has the most amount of data (100 $\mathrm{min}$ ), we also parcellated each CoRR-HNU subject using one or more rs-fMRI sessions and evaluated the resting-state homogeneity with the remaining sessions. This allowed us to estimate how much the various algorithms would improve with more data. When comparing between parcellations, the effect size (Cohen's $d$ ) of differences was computed. It is worth emphasizing that the evaluation utilized new rs-fMRI data not used for estimating the individual-specific parcellations.

- Task functional inhomogeneity. The HCP task-fMRI data consisted of 7 functional domains: social cognition, motor, gambling, working memory, language processing, emotional processing, and relational processing, each with multiple task contrasts (Barch et al., 2013). For a given task contrast, task inhomogeneity was defined as the standard deviation of (activation) z-values within each network (Gordon, Laumann, Gilmore, Newbold, et al. 2017c; Schaefer et al. 2017). A lower standard deviation indicated higher functional homogeneity 
within the network. The standard deviations were averaged across all networks while accounting for network size:

$$
\frac{\sum_{l=1}^{L} s t d_{l}|l|}{\sum_{l=1}^{L}|l|},
$$

where $s t d_{l}$ is the standard deviation of task activation $z$-values for network $l$ and $|l|$ is the number of vertices in parcel $l$ (Gordon, Laumann, Gilmore, Newbold, et al. 2017c; Schaefer et al. 2017).

For each subject in the HCP test set $(N=596)$, the first rs-fMRI run from the first session was used to infer the individualspecific parcellation. The individual-specific parcellation was then utilized to evaluate task inhomogeneity for each task contrast (eq. (2)) and then averaged across all contrasts within a functional domain, resulting in a single functional inhomogeneity measure per functional domain. The number of task contrasts per functional domain ranged from 3 for the emotion domain to 8 for the working memory domain. When comparing between parcellations, the inhomogeneity metric (eq. (2)) was averaged across all contrasts within a functional domain before the effect size (Cohen's d) of differences was computed for each functional domain.

We note that a cortical parcellation with more networks would on average perform better on the proposed evaluation metrics. The reason is that a cortical parcellation with more networks will have smaller networks (on average), resulting in higher connectional homogeneity and lower functional inhomogeneity. For example, if a network consisted of only 2 vertices, then it would be highly homogeneous. However, this was not an issue in our experiments because all approaches were constrained to estimate the same number of networks (i.e., 17 networks). Furthermore, the evaluation metrics (eqs (1) and (2)) accounted for network size, so a network with only 2 vertices would only contribute minimally to the final homogeneity metric.

\section{Intra-subject Reproducibility and Inter-subject Similarity of MS-HBM Network Topography}

Having established that the MS-HBM was better than other approaches in generating individual-specific parcellations, the reproducibility of individual-specific MS-HBM networks was further characterized using the CoRR-HNU data and HCP test set. Given that intra-subject and inter-subject network variabilities were different across networks, we were interested in evaluating whether intra-subject network reproducibility and inter-subject network similarity were also different across networks.

Individual-specific MS-HBM parcellations were independently inferred using the first 2 runs and the last 2 runs of the HCP test set. Therefore, there were 2 individual-specific parcellations for each subject based on data from 2 independent sets of rs-fMRI data. MS-HBM parcellations were also independently inferred using sessions 1-5 and sessions $6-10$ of the CoRR-HNU dataset. Therefore, there were 2 individual-specific parcellations for each subject based on data from 2 independent sets of 5 sessions.

To evaluate the reproducibility of individual-specific parcellations, the Dice coefficient was computed for each network from the 2 parcellations of each subject. The Dice coefficients were then averaged across all networks and all subjects to provide an overall measure of intra-subject parcellation reproducibility. To evaluate inter-subject parcellation similarity, for each pair of subjects, the Dice coefficient was computed for each network. Since there were 2 parcellations for each subject, there were a total of 4 Dice coefficients for each network, which were then averaged. The Dice coefficients were then averaged across all networks and all pairs of subjects to provide an overall measure of inter-subject parcellation similarity.

\section{HCP Behavioral Data}

Given that individual-specific functional networks exhibited unique topographical features not observed in group-level networks, we further investigated whether the spatial configuration of individual-specific cortical parcellations was behaviorally meaningful. Since the HCP dataset has a rich repertoire of behavioral data, we selected 58 behavioral phenotypes measuring cognition, personality and emotion (Supplementary Table S1). Eighteen subjects were excluded from further analyses because they did not have all behavioral phenotypes, resulting in a final set of 577 subjects. Individual-specific MS-HBM parcellations were estimated for each HCP test subject $(N=577)$ using all 4 rs-fMRI runs, where each run was treated as coming from an independent session. We note that very similar parcellations were obtained if we averaged the connectivity profiles across the 2 fMRI runs within each day, treating each day as an independent session.

Because the 58 behavioral measures were correlated, we also considered a subset of 5 minimally correlated behavioral measures. The 5 behavioral measures were selected as follows. We randomly picked a pair of behavioral measures with an absolute correlation of less than 0.1. Three more behavioral measures were added one at a time, while ensuring that each newly added behavioral measure was minimally correlated (absolute $r<0.1$ ) with the current set of behavioral measures. This procedure was repeated 100 times, resulting in 100 sets of 5 behavioral measures. The behavioral set with the smallest maximum absolute correlation was selected. The final set of 5 behavioral measures corresponded to reading (pronunciation), positive affect, grip strength, social cognition (random) and contrast sensitivity (Supplementary Table S1). The maximum absolute correlation was $r=0.068(P=0.104$, which is not significant even if we ignore multiple comparisons).

\section{Can Individual-Specific Network Spatial Topography Be Used to Predict Behavior?}

Kernel regression (Murphy 2012) was utilized to predict each behavioral phenotype in individual subjects. Suppose $y$ is the behavioral measure (e.g., fluid intelligence) and $l$ is the individual-specific parcellation of a test subject. In addition, suppose $y_{i}$ is the behavioral measure (e.g., fluid intelligence) and $l_{i}$ is the individual-specific parcellation of the ith training subject. Then kernel regression would predict the behavior of the test subject as the weighted average of the behaviors of the training subjects: $y \approx \sum_{i \in \text { training set }} \operatorname{Similarity}\left(l_{i}, l\right) y_{i}$, where Similarity $\left(l_{l}, l\right)$ was the Dice overlap coefficient between corresponding networks of the test subject and ith training subject, averaged across 17 networks. Therefore, successful prediction would indicate that subjects with more spatially overlapping networks (i.e., network topography) have similar behavioral measures.

In practice, we included an $l_{2}$-regularization term (i.e., kernel ridge regression) to reduce overfitting (Supplementary Methods S6; Murphy 2012). The $l_{2}$-regularization parameter was determined via an inner-loop cross-validation procedure. More 
specifically, we performed 20-fold cross-validation for each behavioral phenotype. Care was taken so that family members were not split between folds. For each test fold, 20-fold crossvalidation was repeatedly applied to the remaining 19 folds with different regularization parameters (i.e., inner-loop crossvalidation). The optimal regularization parameter from the inner-loop cross-validation was then used to predict the behavioral phenotype in the test fold. Accuracy was measured by correlating the predicted and actual behavioral measure across all subjects within the test fold (Finn et al. 2015). By repeating the procedure for each test fold, each behavior yielded 20 correlation accuracies, which were then averaged across the 20 folds. Because a single 20-fold cross-validation might be sensitive to the particular split of the data into folds (Varoquaux et al. 2017), the above 20-fold cross-validation was repeated 100 times. The mean accuracy and standard deviation across the 100 cross-validations will be reported.

Finally, certain behavioral measures are known to correlate with motion (Siegel et al., 2017). Therefore, age, sex, frame-wise displacement (FD), DVARS, body mass index, and total brain volume were regressed from the behavioral data before kernel ridge regression. To prevent any information leak from the training data to test data, for each test fold, the nuisance regression was performed on the training folds and the regression coefficients were applied to the test fold.

\section{Comparison With Alternative Parcellation Approaches}

The above prediction procedure was repeated using parcellations estimated by YeoBackProject, Gordon2017, and Wang2015. The procedure could not be applied to the Yeo2011 approach because the group-level approach results in the same parcellations across subjects.

\section{Network Size Versus Topography}

In the previous sections, a test subject's behavior was predicted based on the similarity between the individual-specific parcellations of the test subject and the training subjects, where similarity was measured based on how much corresponding networks spatially overlapped (i.e., Dice). Here, we investigated whether individual differences in network size could also predict behavior. This was achieved by defining the similarity between 2 parcellations to be the correlation between network sizes. More specifically, let $s$ and $s_{i}$ be $17 \times 1$ vectors with the jth entries corresponding to the surface areas belonging to the jth network of the test subject and ith training subject, respectively. Here, surface areas were measured in the subjects' native space. The similarity between the individual-specific parcellations of the test subject and ith training subject was set to be the correlation between $s$ and $s_{i}$. Therefore, test subject and ith training subject might be similar due to corresponding networks having similar sizes, even though the networks might not significantly overlap. If the prediction accuracies were significantly higher for network overlap (compared with network size), then this would suggest that network topography (location), and not network size, was driving the behavioral prediction.

\section{Topography of Task-Relevant Networks}

We also investigated whether the topography of task-relevant networks might contribute to the prediction of various behavioral measures. For example, the frontoparietal control network is typically activated during working memory tasks. Would the topography of the individual-specific frontoparietal control network be more predictive of working memory performance than the topography of all networks?

To explore this question in a systematic fashion, we considered 13 cognitive measures highlighted in the HCP data dictionary (first 13 items in Supplementary Table S1). For each cognitive measure, we searched for the "forward inference" map of the most relevant term in the NeuroSynth database (Yarkoni et al. 2011). The forward inference map quantified the likelihood that a particular brain voxel was activated in studies using that search term. The reverse inference maps were not considered because they turned out to be extremely sparse. Supplementary Table S2 shows the search term utilized to obtain the forward inference map for each cognitive measure. There was no appropriate search term for 2 cognitive measures (processing speed and picture vocabulary), so they were excluded from further analyses. Each forward inference map was projected to fs_LR surface space (Buckner et al. 2011; Van Essen, Glasser, et al. 2012a) and compared with the group-level parcellation estimated from the HCP training set, in order to select the task-relevant networks (Supplementary Table S2). When predicting a particular behavior, the similarity between the test subject and ith training subject was set to be the dice coefficient averaged across task-relevant networks. For example, in the case of fluid intelligence, Control network A and Dorsal Attention network A overlapped the most with the forward inference map associated with "intelligence" (Supplementary Table S2). When applying kernel regression to predict fluid intelligence, similarity between the test subject and ith training subject was set to be the average dice coefficient for Control network A and Dorsal Attention network A.

\section{Code Availability}

Code for this work is freely available at the github repository maintained by the Computational Brain Imaging Group (https:// github.com/ThomasYeoLab/CBIG). More specifically, the GSP and CoRR-HNU datasets were preprocessed using an in-house pipeline (https://github.com/ThomasYeoLab/CBIG/tree/master/ stable_projects/preprocessing/CBIG_fMRI_Preproc2016). The group-level parcellation code (Yeo et al. 2011) is available here (https://github.com/ThomasYeoLab/CBIG/tree/master/stable_ projects/brain_parcellation/Yeo2011_fcMRI_clustering). Finally, the individual-specific parcellation code is also available (https:// github.com/ThomasYeoLab/CBIG/tree/master/stable_projects/brain parcellation/Kong2019_MSHBM)

\section{Results}

\section{Overview}

The MS-HBM (Fig. 1) was applied to 3 multi-session rs-fMRI datasets to ensure that the model can reliably estimate intersubject and intra-subject variability despite significant acquisition differences across datasets. After confirming the previous literature (Mueller et al. 2013; Laumann et al. 2015) that intersubject and intra-subject RSFC variabilities were different across networks, we then established that the MS-HBM produced better parcellations than other approaches. Finally, we investigated whether the topography (location and spatial arrangement) and size of individual-specific cortical networks were behaviorally relevant. 
A Group parcellation
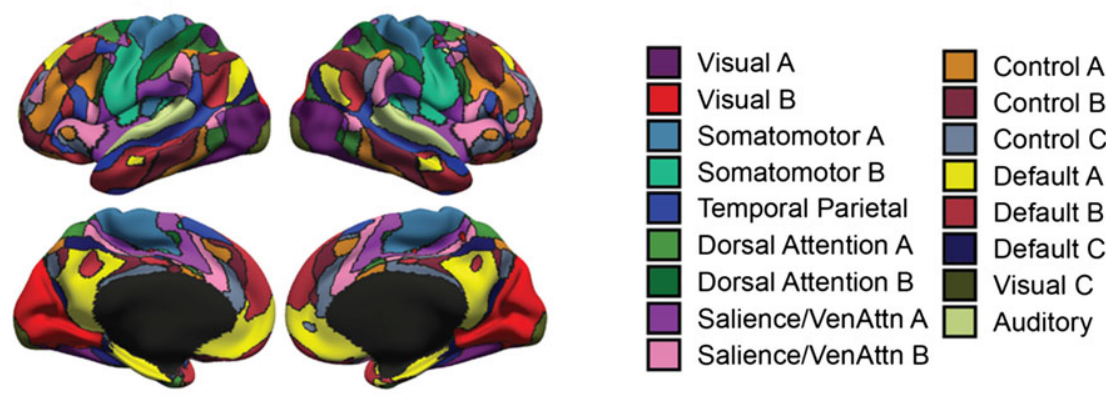

B Inter-subject RSFC variability

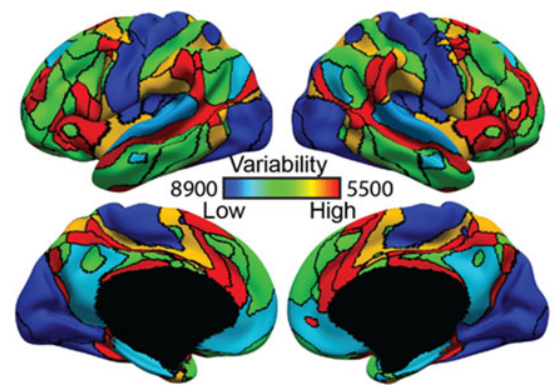

C Intra-subject RSFC variability

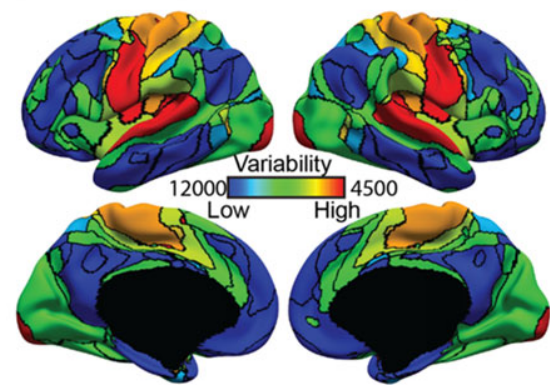

Figure 2. Sensory-motor networks exhibit lower inter-subject, but higher intra-subject, functional connectivity variability than association networks in the HCP training set. (A) 17-network group-level parcellation. (B) Inter-subject functional connectivity variability for different cortical networks. (C) Intra-subject functional connectivity variability for different cortical networks. Results were replicated in the GSP (Supplementary Fig. S1) and CoRR-HNU (Supplementary Fig. S2) datasets. Note that (B) and $(C)$ correspond to the $\epsilon_{l}$ and $\sigma_{l}$ parameters in Figure 1, where higher values indicate lower variability.

\section{Sensory-Motor Networks Exhibit Lower Inter-subject, but Higher Intra-subject, Functional Connectivity Variability Than Association Networks}

Figure 2A shows the 17-network population-level parcellation estimated from the HCP training set. The 17 networks were divided into 8 groups (Visual, Somatomotor, Auditory, Dorsal Attention, Salience/Ventral Attention, Control, Default, and TempPar), which broadly corresponded to major networks discussed in the literature. The 17 networks were referred to as "Default A," "Default B," and so on (Fig. 2A).

The HCP population-level parcellation was replicated in the GSP (Supplementary Fig. S1A) and CoRR-HNU (Supplementary Fig. S2A) datasets, although there were some interesting distinctions. For example, the Limbic (A and B) networks from the GSP population-level parcellation (Supplementary Fig. S1A) were absorbed into the Default (A and B) networks in the HCP population-level parcellation (Fig. 2A). Instead, there were 2 additional networks in the HCP population-level parcellation: Visual $\mathrm{C}$ and Auditory networks. The Visual C network (Fig. 2A) might correspond to the foveal representation within the primary visual cortex, while the Auditory network (Fig. 2A) appeared to have split off from the Somatomotor B network in the GSP population-level parcellation (Supplementary Fig. S1A).

Increasing the number of subjects only resulted in minor changes in the group-level parcellations, so differences between population-level parcellations were probably due to acquisition differences, rather than sampling variability. For example, the higher resolution HCP data might allow the separation of the Auditory and Somatomotor B networks, which were in close spatial proximity.

Recall that the inter-subject functional connectivity variability $\epsilon_{l}$ was estimated for each network. Hence, $\epsilon_{l}$ could be visualized by coloring each corresponding population-level network from Figure $2 A$. Figure $2 B$ shows $\epsilon_{l}$ estimated from the HCP training set. Consistent with previous literature (Laumann et al. 2015), sensory-motor networks exhibited lower inter-subject functional connectivity variability than association networks. More specifically, Somatomotor (A and B) and Visual (A and $B$ ) networks were the least variable, while Salience/Ventral Attention B network was the most variable. The results were largely consistent in the GSP (Supplementary Fig. S1B) and CoRR-HNU (Supplementary Fig. S2B) datasets, although there were some notable differences. For example, the Somatomotor B network exhibited low variability in both the GSP and HCP datasets, but intermediate variability in the CoRR-HNU dataset.

Similar to $\epsilon_{l}$, the intra-subject functional connectivity variability $\sigma_{l}$ was estimated for each network. Hence, $\sigma_{l}$ could be visualized by coloring each corresponding population-level network from Figure 2A. Figure 2C shows $\sigma_{l}$ estimated from the HCP training set. Consistent with previous literature (Laumann et al. 2015), association networks exhibited lower intra-subject functional connectivity variability than sensory-motor networks. More specifically, Default (A and B) networks were the least variable, while Somatomotor (A and B), Auditory and Visual C networks were the most variable. The results were largely consistent in the GSP (Supplementary Fig. S1C) and CoRR-HNU (Supplementary Fig. S2C) datasets, although there were some interesting differences. Of particular note is that Visual B Network exhibited high intra-subject functional connectivity variability in the GSP dataset, but low or intermediate functional connectivity variability in the CoRR-HNU and HCP datasets. This difference might be due to subjects instructed to fixate on a cross in the CoRR-HNU and HCP datasets, while subjects were told to keep their eyes open (with no fixation cross) in the GSP dataset. 
It is worth noting that in the MS-HBM (Fig. 1), higher values of $\epsilon_{l}$ and $\sigma_{l}$ indicate lower variability. The values in Figure $2 \mathrm{C}$ are much larger than Figure $2 B$, suggesting that intra-subject functional connectivity variability is much lower than intersubject functional connectivity variability. These results were replicated in the GSP (Supplementary Fig. S1) and CoRR-HNU (Supplementary Fig. S2) datasets.

\section{Sensory-Motor Networks Are Less Spatially Variable Than Association Networks Across Subjects}

The MS-HBM model differentiated between inter-subject RSFC and network spatial variability. Like inter-subject functional connectivity variability, the sensory-motor networks were found to be less spatially variable than association networks across subjects. For example, Supplementary Figure S3 shows the inter-subject spatial variability maps of 4 representative networks from the HCP training set. Yellow color at a spatial location indicates that across subjects, there is a high probability of the network appearing at that spatial location, suggesting low inter-subject spatial variability. The Somatomotor A network and Visual B network showed higher probabilities (more yellow color) than the Dorsal Attention networks, suggesting that Somatomotor A network and Visual B network exhibited lower inter-subject spatial variability than Dorsal Attention networks. These results were consistent in the GSP (Supplementary Fig. S4) and CoRR-HNU (Supplementary Fig. S5) datasets.

\section{Individual-Specific Networks Generated by MS-HBM Exhibit Higher Resting-State Homogeneity Than Other Approaches}

Individual-specific parcellations were estimated using one rs-fMRI session from the CoRR-HNU dataset and HCP test set. The resting-state homogeneity of the parcellations was evaluated in the leave-out sessions (Fig. 3A). Across both CoRR-HNU and HCP datasets, the group-level parcellation (Yeo2011) achieved the worst resting-state homogeneity, while MS-HBM performed the best. In the CoRR-HNU dataset, compared with Yeo2011, YeoBackProject, Gordon2017, and Wang2015, the MS-HBM achieved an improvement of 16.6\% (Cohen's $d=4.6$ ), 5.3\% (Cohen's $d=3.5$ ), $6.9 \%$ (Cohen's $d=3.4$ ), and 4.2\% (Cohen's $d=3.4$ ), respectively. In the HCP dataset, compared with Yeo2011, YeoBackProject, Gordon2017, and Wang2015, the MS-HBM achieved an improvement of 9.8\% (Cohen's $d=3.2$ ), 9.5\% (Cohen's $d=3.0$ ), 5.7\% (Cohen's $d=2.1$ ), and $4.4 \%$ (Cohen's $d=3.1$ ), respectively.

Individual-specific parcellations were estimated with increasing number of rs-fMRI sessions using the CoRR-HNU dataset. The resting-state homogeneity of the parcellations was evaluated in the leave-out sessions (Fig. 3B). Not surprisingly, performance of the Yeo2011 group-level parcellation remained constant regardless of the amount of data. The remaining approaches (YeoBackProject, Gordon2017, Wang2015, and MS-HBM) exhibited higher homogeneity with increased number of sessions. Critically, the improvement of our model over the other approaches grew with the inclusion of additional fMRI sessions. For example, as the number of sessions was increased from 2-3 to 4-5, the MS-HBM achieved improvement of $5.4 \%, 5.9 \%, 6.1 \%$, and $6.4 \%$, respectively, over Gordon2017. Interestingly, the improvement of our approach over Gordon2017 was largest when only one rs-fMRI session was utilized (6.9\%). On the other hand, the MS-HBM achieved improvement of $3.6 \%, 4.4 \%, 4.8 \%$, and $5.0 \%$, respectively, over Wang2015. Furthermore, using just one fMRI sessions (10 min), the MS-HBM was able to match the homogeneity achieved with the Wang2015 and Gordon2017 approaches using 5 fMRI sessions (50 $\mathrm{min})$.

\section{Individual-Specific Networks Generated by the MS-HBM Exhibit Lower Task Functional Inhomogeneity Than Other Approaches}

Individual-specific parcellations were estimated using one rs-fMRI run $(15 \mathrm{~min})$ from the HCP test set. Supplementary Figure S6 shows the task inhomogeneity of the different approaches.

Compared with Yeo2011, the MS-HBM achieved a small improvement of $0.63 \%$ (Cohen's $d=0.12,0.09,0.66,1.0,0.9,1.1$, and 0.46 for social, motor, gambling, relational, language, working memory, and emotion, respectively). Compared with YeoBackProject, Gordon2017, and Wang2015, MS-HBM achieved improvements of 2.0\% (Cohen's $d>1.3$ for all domains), $1.04 \%$ (Cohen's $d>0.99$ for all domains), and 0.7\% (Cohen's $d>0.79$ for all domains), respectively. Interestingly, the Yeo2011 grouplevel parcellation performed as well as (or even better than) YeoBackProject and Gordon2017.

\section{Individual-Specific MS-HBM Parcellations Exhibit High Intra-subject Reproducibility and Low Inter-subject Similarity}

To assess intra-subject reproducibility and inter-subject similarity, our model (Fig. 1) was tuned on the HCP training and validation sets, and then applied to the HCP test set. Individual-specific parcellations were generated by using the first 2 runs and last 2 runs separately for each subject. Figure 4 and Supplementary Figure S7 show the parcellations of 4 representative subjects. The 17 networks were present in all individual-specific parcellations. However, network shapes, sizes, and topologies were varied across subjects, consistent with previous studies of individualspecific brain networks (Harrison et al. 2015; Laumann et al. 2015; Wang et al. 2015; Gordon, Laumann, Gilmore, Newbold, et al. 2017c; Braga and Buckner 2017).

For example, the Default A (yellow) network exhibited a lateral temporal component in certain subjects (blue arrows in Fig. 4), but was missing in other subjects. As another example, the 2 lateral prefrontal components of the Control A (orange) network (Fig. 2A) were fused into a single component in certain subjects (green arrows in Fig. 4). These features were mostly replicated across sessions. Examples from the CoRR-HNU dataset are shown in Supplementary Figures S8 and S9.

Supplementary Figure S10A shows the across-subject spatial similarity (Dice coefficient) of individual-specific parcellations. A higher value (hot color) indicates greater inter-subject agreement. Supplementary Figure S10B shows the within-subject reproducibility (Dice coefficient) of individual-specific parcellations. A higher value (hot color) indicates greater intersession agreement within subjects. Further quantification is shown in Supplementary Figure S10C, where the Dice coefficients were averaged across sub-networks.

Across all networks, intra-subject reproducibility was greater than inter-subject similarity. Compared with association networks, the Somatomotor (A and B) networks and Visual (A and B) networks were more spatially similar across subjects, but also exhibited greater within-subject intersession reproducibility. Overall, the MS-HBM parcellation model achieved $77.9 \%$ intrasubject reproducibility and $65.4 \%$ inter-subject similarity.

The results were similar in the CoRR-HNU dataset (Supplementary Fig. S11), although intra-subject reproducibility 

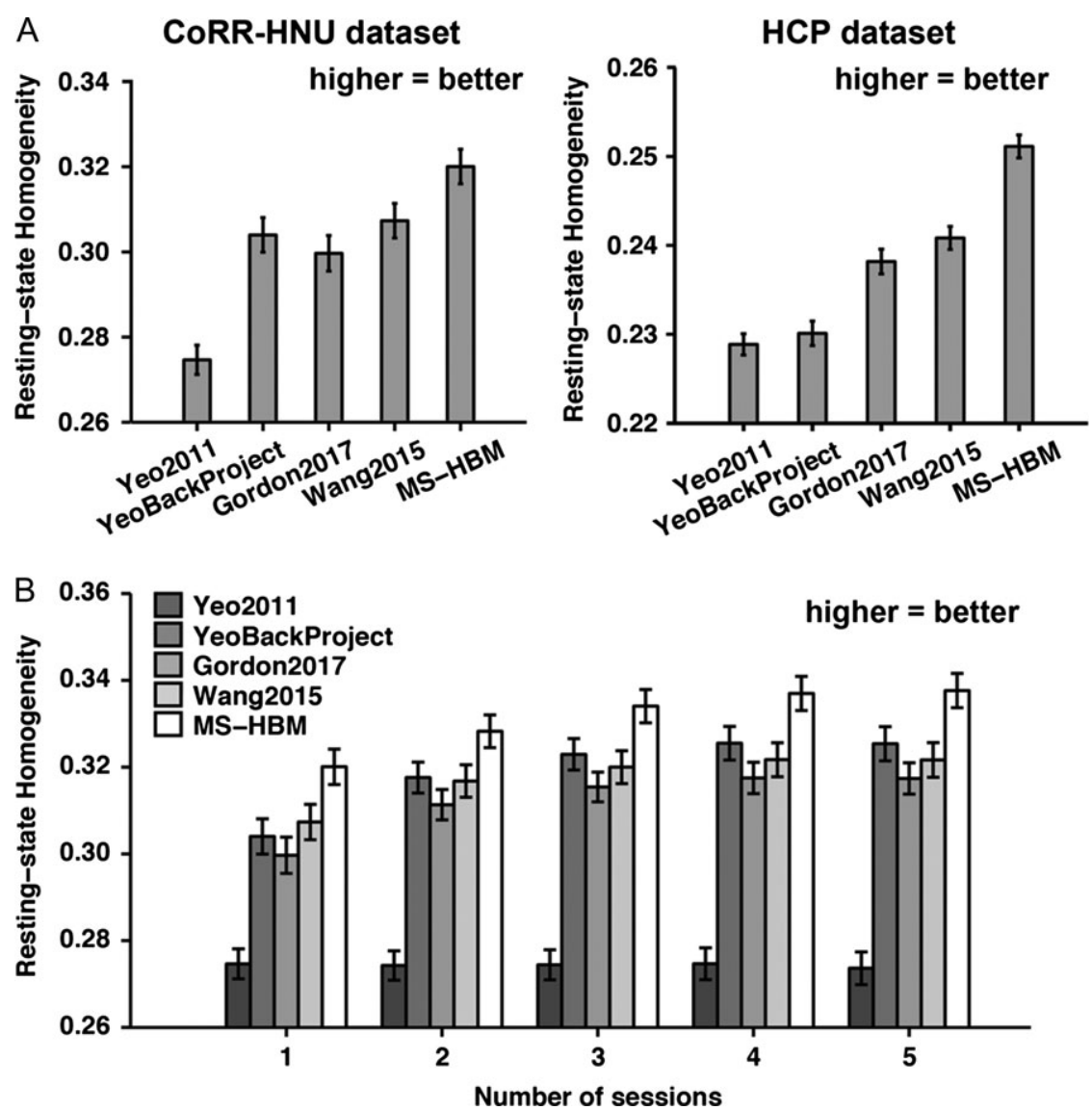

Figure 3. Resting-state homogeneity in the CoRR-HNU and GSP dataset. (A) 17-network individual-specific parcellations were estimated using one rs-fMRI session and resting-state homogeneity were computed on the remaining sessions for each subject from the CoRR-HNU and HCP dataset. (B) 17-network individual-specific parcellations were estimated using different number of rs-fMRI sessions and resting-state homogeneity were computed on the remaining sessions for each subject from the CoRR-HNU dataset. Error bars correspond to standard errors. Using just one single fMRI sessions (10 min), the MS-HBM algorithm was able to match the homogeneity achieved with Wang2015 and Gordon2017 using 5 fMRI sessions (50 min).
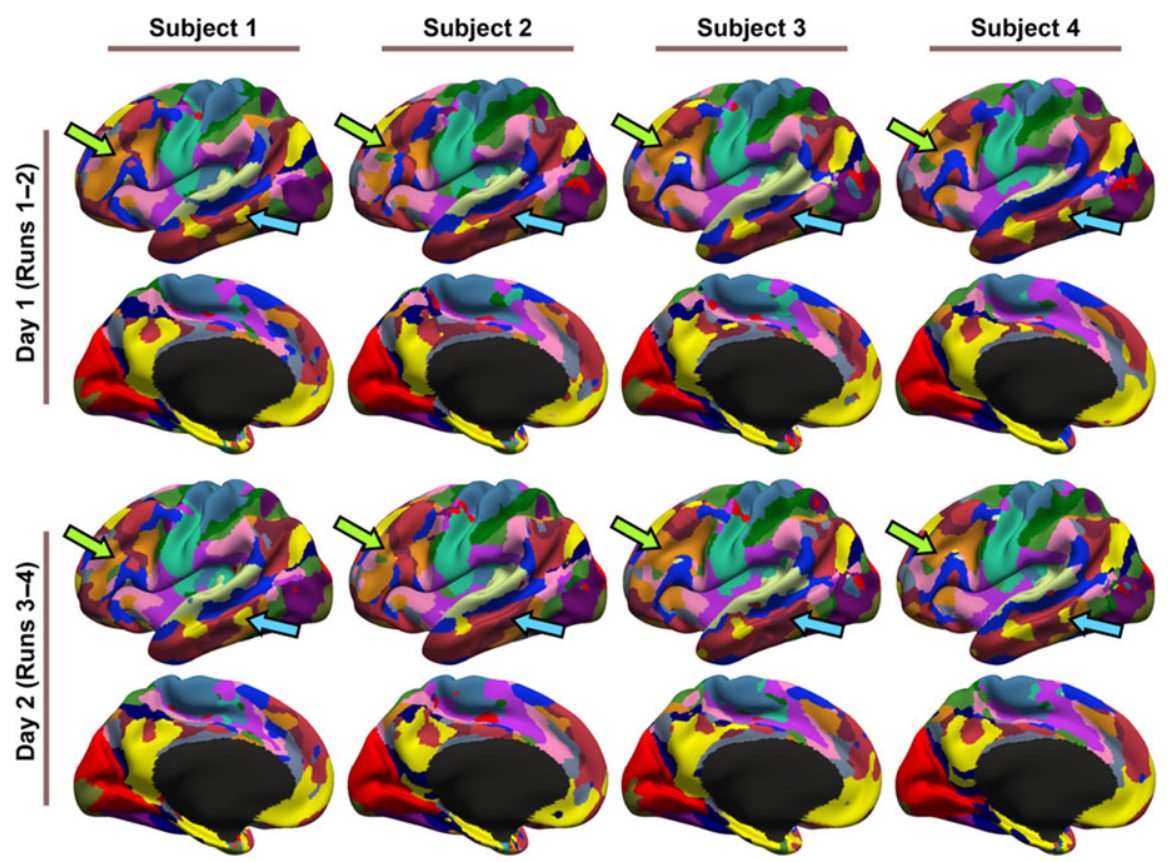

Figure 4. 17-network parcellations were estimated using runs 1-2 and runs 3-4 separately for each subject from the HCP test set. Parcellations of 4 representative subjects are shown here. Blue and green arrows indicate individual-specific parcellation features. Right hemisphere parcellations are shown in Supplementary Figure S7. 
was higher (81.6\%) and inter-subject similarity was lower (59.4\%). The improvement might be the result of longer scan duration in the CoRR-HNU dataset ( $50 \mathrm{~min}$ vs. $30 \mathrm{~min}$ ).

\section{Individual Differences in Network Topography Can Predict Cognition, Personality, and Emotion}

While it is well known that individual-specific networks exhibit unique topographic features that are replicable across sessions (Laumann et al. 2015; Gordon, Laumann, Gilmore, Newbold, et al. 2017c; Braga and Buckner 2017), their behavioral relevance remains unclear. Here, we found that individual-specific network topography was able to predict the 58 behavioral measures with varying degree of accuracies.

Figure 5 shows the prediction accuracy for 13 cognitive measures highlighted in the HCP data dictionary. Average prediction accuracy was $r=0.1321 \pm 0.0053$. Reading (pronunciation) and delay discounting could be predicted relatively well with accuracies of $r=$ $0.2918 \pm 0.0141$ (mean \pm std) and $r=0.2398 \pm 0.0166$. The prediction accuracies for the remaining cognitive, emotion, and personality measures are found in Supplementary Figures S12 and S13. In the case of the NEO-5 personality scores (Supplementary Fig. S12), average predication accuracy was $r=0.0955 \pm 0.0085$. Interestingly, the prediction of emotional recognition (Supplementary Fig. S13) was poor with an average prediction accuracy of $r=-0.0445 \pm 0.0101$. The remaining emotional measures (all items in Supplementary Fig. S13 except for emotional recognition) could be predicted with an average accuracy of $r=0.1038 \pm 0.0070$.

In the case of the minimally correlated set of 5 behavioral measures, the average prediction accuracy was $r=0.1327 \pm$ 0.0065 . Across all 58 behavioral measures, an average prediction accuracy of $r=0.0803 \pm 0.0032$ (mean \pm std) was obtained. While the accuracy might seem modest, they were comparable to (if not better than) other studies using functional connectivity strength for behavioral prediction (HCP MegaTrawl; https://

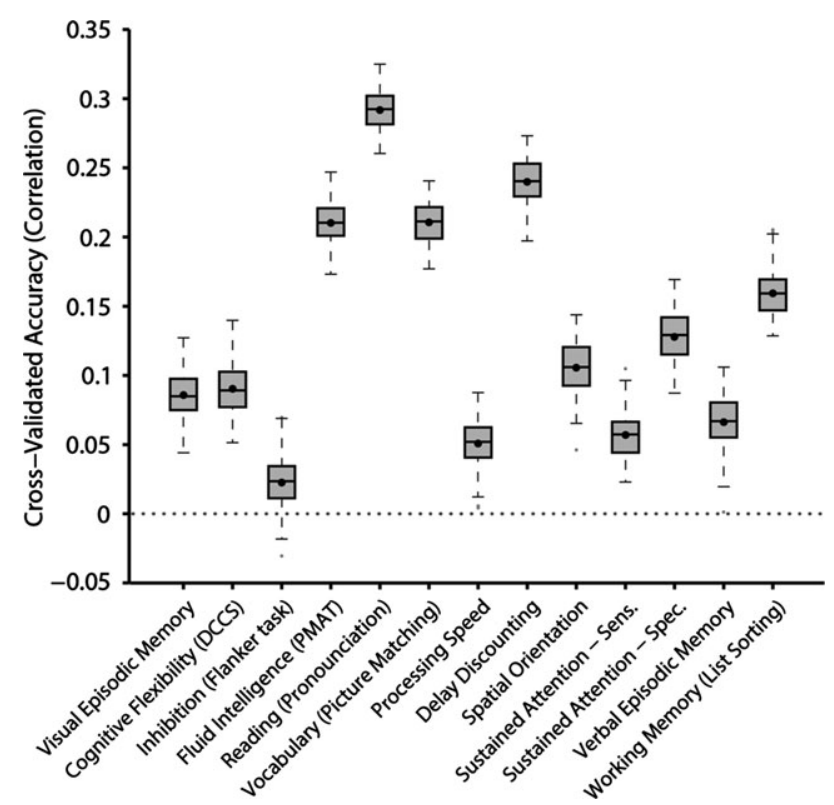

Figure 5. Prediction accuracy of 13 cognitive measures based on inter-subject differences in the spatial arrangement of cortical networks. Boxplots utilized default Matlab parameters, i.e., box shows median and interquartile range (IQR). Whiskers indicate 1.5 IQR. Dot indicates mean. Average prediction accuracy was $r=0.1321 \pm 0.0053$ (mean \pm std) for the 13 measures. Other behavioral measures are found in Supplementary Figures S12 and S13. db.humanconnectome.org/megatrawl/; Dubois et al. 2017; Noble et al. 2017). For example, of the 58 behavioral measures, 49 of them were also utilized in the HCP MegaTrawl. For the 300-dimensional group-ICA results, HCP MegaTrawl achieved an average accuracy of $r=0.0592$ (original data space), while kernel regression yielded an average accuracy of $r=0.0874 \pm$ 0.0036 .

\section{MS-HBM Network Topography Is More Predictive of Behavioral Measures Compared With Other Parcellation Approaches}

Supplementary Table S3 summarizes the average prediction accuracies for different sets of behavioral measures ( 58 behavioral measures, 13 cognitive measures, NEO-5 personality measures, emotion recognition measures, emotional measures, and minimally correlated set of 5 behaviors). Overall, MS-HBM network topography achieved better prediction accuracies compared with other approaches.

Figure 6 shows the average prediction accuracies of the minimally correlated behavioral set across different parcellation approaches. Compared with YeoBackProject, Gordon2017, and Wang2015, MS-HBM achieved percentage improvements of $29 \%, 61 \%$, and $28 \%$, respectively. Furthermore, MS-HBM achieved the best prediction accuracy for the minimally correlated behavioral set for each of the 10020 -fold cross-validations (Supplementary Table S3).

There were 2 exceptions. First, MS-HBM performed the worst in the emotional recognition measures. Second, network topography estimated with YeoBackProject was better than MS-HBM in predicting NEO-5 personality measures.

\section{Controlling for Motion-Related Imaging Artifacts}

Given that certain behavioral measures are known to correlate with motion (Siegel et al., 2017), we tested if network

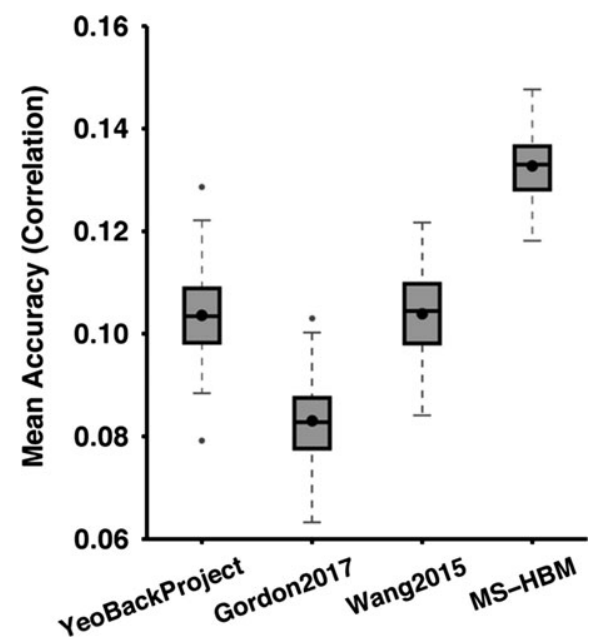

Figure 6. Average prediction accuracies of the minimally correlated set of 5 behavioral measures across different parcellation approaches. Prediction was based on individual-specific network topography. Boxplots utilized default Matlab parameters, i.e., box shows median and interquartile range (IQR). Whiskers indicate 1.5 IQR. Dot indicates mean. MS-HBM, YeoBackProject, Gordon2017, and Wang2015 achieved average prediction accuracies of $r=$ $0.1327 \pm 0.0065$ (mean \pm std), $r=0.1036 \pm 0.0080, r=0.0830 \pm 0.0080$ and $r=$ $0.1039 \pm 0.0080$. 
topography could predict FD and DVARS (without regressing any nuisance covariates). Network topography estimated by all 4 parcellation approaches (MS-HBM, YeoBackProject, Gordon2017, and Wang2015) could predict FD and DVARS quite well (Supplementary Table S4), suggesting that individualspecific network topography might encode information about motion-related imaging artifacts or motion-related traits or both (Zeng et al. 2014; Siegel et al. 2017).

If individual-specific MS-HBM parcellations were corrupted by motion-related imaging artifacts, then state-related FD and DVARS would have a significant effect on intra-subject parcellation reproducibility. However, further analyses involving participants with very different FD and DVARS across the 2 scan days indicated that state-related FD and DVARS had little effect on intra-subject reproducibility (Supplementary Figs S14 and S15). Together with the successful prediction of FD and DVARS (Supplementary Table S4), this suggests that individual-specific network topography (estimated by MS-HBM) likely encoded a significant amount of information about motion-related traits. Nevertheless, we cannot completely rule out the possibility that aspects of network topography might be corrupted by motion-related imaging artifacts.

To address the concern that the behavioral prediction results in the previous sections might simply be due to the regression algorithm encoding motion-related imaging artifacts that were correlated with the behavioral measure of interest, we considered the 5 behavioral measures most correlated with FD: endurance, cognitive flexibility (DCCS), vocabulary (picture matching), and reading (pronunciation). Supplementary Figure S16A shows that the average correlation between the prediction of the 5 behavioral measures and FD was close to zero, and was significantly lower than the average prediction accuracy of the 5 behavioral measures. In fact, across the 58 behavioral measures, higher correlation with FD was associated with worse behavioral prediction accuracy $(r=-0.22)$. Similar results were obtained for DVARS (Supplementary Fig. S16B). Together, this suggests that the behavioral prediction results in the previous section could not be simply explained by motion-related imaging artifacts.

\section{Network Size Versus Network Topography}

When utilizing individual differences in network size to predict behavior, the average accuracy of the minimally correlated set of 5 behavioral measures was $r=0.0865 \pm 0.0105$ (mean \pm std; Fig. 7). This was worse than using network topography (Fig. 7). Average prediction accuracy for the 13 cognitive measures was $r=0.0483 \pm 0.0073$. In the case of the NEO-5 personality scores, average prediction accuracy was $r=0.0751 \pm 0.0100$, while the average prediction accuracy of the emotional measures was $r=$ $0.0782 \pm 0.0086$. The average prediction accuracy of 58 behavioral measures was $r=0.0412 \pm 0.0047$. Thus, individual differences in network size (at least at the resolution of large-scale networks) could not account for the ability of network topography to predict behavior.

\section{Topography of Task-Relevant Networks}

When utilizing the topography of only task-relevant networks (rather than all networks) to predict behavior, the average accuracy across 11 cognitive measures (see Methods) was $r=0.1129$ \pm 0.0062 (mean \pm std; Fig. 8). This was worse than utilizing the topography of all networks $r=0.1324 \pm 0.0056$.

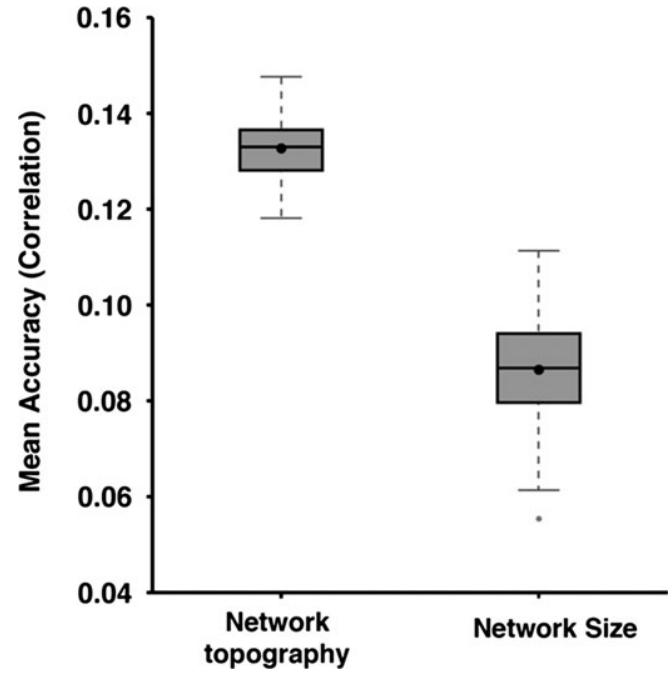

Figure 7. Average prediction accuracies of the minimally correlated set of 5 behavioral measures based on inter-subject differences in network topography or network size. Boxplots utilized default Matlab parameters, i.e., box shows median and interquartile range (IQR). Whiskers indicate 1.5 IQR. Dot indicates mean. Average prediction accuracy based on network topography was $r=$ $0.1327 \pm 0.0065$ (mean \pm std). Average prediction accuracy based on network size was $r=0.0865 \pm 0.0105$.

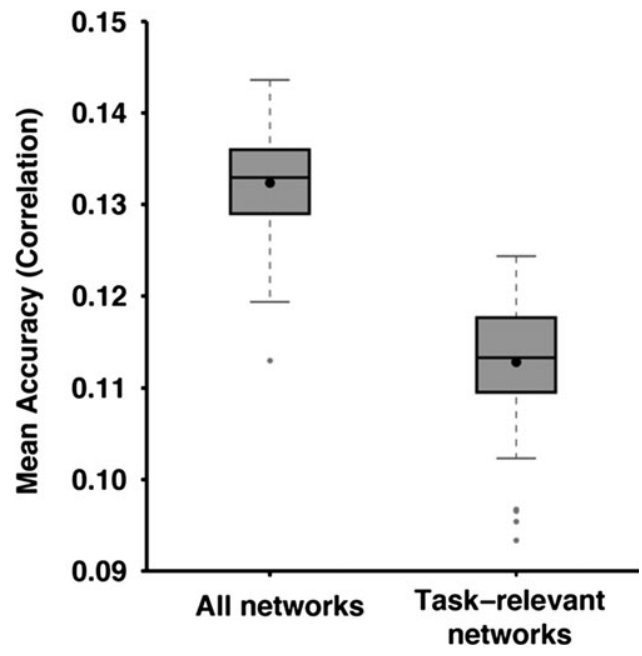

Figure 8. Average prediction accuracy across 11 cognitive measures based on topography of all networks or only task-relevant networks. Boxplots utilized default Matlab parameters, i.e., box shows median and interquartile range (IQR). Whiskers indicate 1.5 IQR. Dot indicates mean. Average prediction accuracy based on all networks was $r=0.1324 \pm 0.0056$. Average prediction accuracy based only on task-relevant networks was $r=0.1129 \pm 0.0062$. Prediction accuracy of each cognitive measure is found in Supplementary Figure S17.

\section{Discussion}

Using a novel MS-HBM, we demonstrate that individuallyspecific cortical network topography could predict multiple behavioral phenotypes across cognition, personality and emotion. The prediction accuracies could not be accounted for by individual differences in network size. The MS-HBM allowed the joint estimation of inter-subject variability, intra-subject variability and individual-specific cortical networks within the same statistical framework. The resulting MS-HBM individualspecific parcellations were more homogeneous than parcellations derived with 4 alternative approaches during both resting 
and task states. These analyses suggest that the spatial topography of individuals-specific brain networks might serve as a generalizable fingerprint of human behavior, similar to the preponderance of studies utilizing functional connectivity strength.

\section{Neurobiological Interpretation of MS-HBM Networks}

By assuming individual-specific parcellations to be the same across sessions, the MS-HBM essentially treats intersession differences as noise. The implication is that individual-specific MS-HBM parcellations seek to capture stable, trait-like network organization in individuals. However, it is well known that certain factors (e.g., caffeine intake, sleepiness, attention) result in different brain states and thus functional network organization (Tagliazucchi and Laufs 2014; Laumann et al. 2015; Poldrack et al. 2015; Yeo, Tandi, et al. 2015b; Shine et al. 2016; Wang et al. 2016). Moreover, in longitudinal studies of certain populations, e.g., Alzheimer's Disease dementia, the goal is to detect longitudinal changes across consecutive sessions (Misra et al. 2009; Raj et al. 2015; Risacher et al. 2010; Zhang et al. 2016; Lindemer et al. 2017). To capture transient session-specific or longitudinal changes in brain network organization, the model could be modified to allow for spatial differences in individualspecific parcellations across sessions.

The human cerebral cortex is hierarchically organized (Churchland and Sejnowski 1988) from molecules (1 Å) to synapses $(1 \mu \mathrm{m})$ to neurons $(100 \mu \mathrm{m})$ to areas $(1 \mathrm{~cm})$ and systems $(10 \mathrm{~cm})$. Even at the relatively coarse spatial resolution of MRI, hierarchical organization can be observed. Here, we focused on parcellating the cerebral cortex into less than twenty spatially distributed networks. Each spatial (e.g., parietal) component of a network likely spans multiple cytoarchitectonically, functionally and connectionally distinct cortical areas (Kaas 1987; Felleman and Van Essen 1991; Amunts and Zilles 2015; Eickhoff et al. 2018). We are working on extending the MS-HBM to estimate a finer division of the cerebral cortex that approximate these finer regions, i.e., individual-specific areal-level parcellation (Laumann et al. 2015; Glasser et al. 2016; Gordon, Laumann, Gilmore, Newbold, et al. 2017c).

A biologically plausible individual-specific parcellation should simultaneously capture (genuinely) shared features across individuals, while preserving individual-specific traitlevel variation. All individual-specific parcellation approaches ultimately make explicit or implicit assumptions about what features are shared or varied across individuals. For example, in the MS-HBM, an important parameter is the number of networks. While there is no penalty for a participant to exhibit a smaller number of networks, in practice, we do find all networks in all subjects. One could potentially estimate different number of networks in each participant by parcellating each participant independently (Laumann et al. 2015; Gordon, Laumann, Gilmore, Newbold, et al. 2017c). However, given the significantly less data available in each subject (compared with Gordon, Laumann, Gilmore, Newbold, et al. 2017c), any network differences between subjects (e.g., less or more networks) could simply be due to convergence to different local optima as a result of noise.

It is also worth noting that although Gordon and colleagues did not explicitly constrain the number of networks to be the same in each participant (Laumann et al. 2015; Gordon, Laumann, Gilmore, Newbold, et al. 2017c), certain parameters that could dramatically affect the estimated networks (e.g., range and sampling intervals of edge density thresholds) were assumed to be the same across subjects. This is a key challenge for all individual-specific parcellation approaches: it is not possible to set completely different parameters for every single individual, since it is unclear how those might be set, and we would also like the parcellations to be comparable across individuals. Yet, setting the same parameters for every individual might not be biologically plausible.

\section{Spatial Configuration of Individual-Specific Cortical Networks Is Behaviorally Meaningful}

Recent work has suggested that individual-specific functional networks exhibit unique topological features not observed in group-level networks (Harrison et al. 2015; Laumann et al. 2015; Wang et al. 2015; Glasser et al. 2016; Langs et al. 2016; Braga and Buckner 2017; Gordon, Laumann, Adeyemo, Petersen, et al. 2017a; Gordon, Laumann, Adeyemo, Gilmore, et al. 2017b; Gordon, Laumann, Gilmore, Newbold, et al. 2017c). This was also clearly the case with individual-specific MS-HBM parcellations (Fig. 4 and Supplementary Fig. S8). While we have pointed out 2 examples (Default A and Control A networks), it was also obvious that many other individual-specific parcellation features were replicable across sessions.

A major unanswered question in the literature is whether individual differences in cortical parcellations are behaviorally meaningful. A recent paper (Salehi et al. 2018) found that individual-specific brain parcellations could be used to predict individuals' sex, while another paper (Bijsterbosch et al. 2018) has associated individual-specific network topography with a single positive-negative axis of behavior. In contrast, we utilized kernel regression to investigate whether individualspecific network topography and size could be used to predict 58 behavioral measures. The kernel regression framework utilized in this work tested the possibility that subjects with more similar parcellations exhibited similar behavior. Successful prediction (Fig. 5, Supplementary Figs S12 and S13) suggests that inter-subject variation in the spatial configuration of cortical networks is strongly related to inter-subject variation in behavior.

The topography of task-relevant networks was also effective for predicting cognitive performance, although interestingly, the prediction accuracies were no better than when using the topography of all networks. It might seem somewhat counterintuitive that the inclusion of task-irrelevant networks (as measured by meta-analysis of task activation) did not dilute prediction accuracy. However, previous studies have suggested that regions not activated by a task might nevertheless exhibit connectivity changes during task performance (Cole et al. 2014; Krienen et al. 2014). Therefore, some of the task-irrelevant networks might potentially be involved in executing a task, despite not being activated through traditional task contrast or subtraction analyses. Alternatively, non-task regions might provide predictive information about traits associated with task performance without direct involvement in the task mechanisms.

Finally, a vast body of literature has utilized inter-region functional connectivity strength to predict behavior (Hampson et al. 2006; Finn et al. 2015; Smith et al. 2015; Yeo, Tandi, et al. 2015b; Rosenberg et al. 2016), in some cases implicitly, e.g., by binarizing a functional connectivity matrix and then utilizing the resulting graph metrics for behavioral association (van den Heuvel et al. 2009). It is worth noting that while spatial topography and functional connectivity strength might seem conceptually orthogonal (Bijsterbosch et al. 2018), they are not trivial to separate in practice. After all, most (if not all) individual- 
specific parcellation (or network estimation) approaches rely to some extent on functional connectivity strength. Therefore, although it seems plausible that network topography estimated by MS-HBM contains neural information complementary to that of functional connectivity strength, some aspects of network topography might still reflect functional connectivity strength.

Consequently, we believe that it will be a worthwhile endeavor to investigate whether individual differences in network topography and individual differences in functional connectivity strength could be combined to further improve behavioral prediction. We note that because only 17 networks were estimated in this work, we could only obtain $17 \times 17$ connectivity matrices, which were not optimal for behavioral prediction (e.g., Finn et al. 2015). We are currently developing an individual-specific areal-level parcellation approach, which would allow us to more effectively explore the possibility of combining network topography and connectivity strength.

\section{Network Size Versus Network Topography}

It is well known that the amount of brain "real estate" devoted to a cognitive function often predicts functional importance or capability. For example, larger hippocampal size has also been associated with better memory (Erickson et al. 2011). As another example, the acquisition of reading skills coincides with a change in the sizes of functionally defined visual areas (Dehaene et al. 2010).

Consistent with the vast literature, our results suggest that individual differences in network size can predict human behavior (Fig. 7). However, the prediction accuracy is significantly weaker than network topography. This is surprising given that there is little evidence in the literature that the location of functional areas or networks might be functionally important.

One important caveat is that most previous studies associating anatomical size with functional importance are focused on smaller brain structures (Erickson et al. 2011; Holmes et al. 2016; Sabuncu et al. 2016), rather than large-scale networks. Therefore, it is possible that prediction accuracies could significantly improve with individual-specific areal-level parcellations.

\section{Individual-specific MS-HBM parcellations are more homogeneous than other approaches during resting and task states}

If an individual-specific parcellation is capturing the organization of a subject's cerebral cortex, then to the first order approximation, one might expect regions within the same network to have similar resting-state time series, as well as similar activation amplitude for any given task contrast (Schaefer et al. 2017; Gordon, Laumann, Gilmore, Newbold, et al. 2017c). Across the CoRR-HNU and HCP datasets, individual-specific MS-HBM parcellations exhibited greater RSFC homogeneity than parcellations from 4 other approaches (Fig. 3), suggesting that MS-HBM parcellations better capture the "intrinsic" organization of individuals' cerebral cortex. Importantly, group-level priors (e.g., inter-subject and intra-subject variability) estimated from the GSP dataset could improve the estimation of individual-specific parcellations in the CoRR-HNU dataset (Fig. 3A). This is important because estimates of inter-subject and intra-subject functional connectivity variability were similar, but not the same across datasets (Fig. 2 and Supplementary Figs S1 and S2). Therefore, our results suggest that the MS-HBM approach can be used to parcellate individuals from new datasets (using the same preprocessing pipeline), without having to re-estimate the group-level priors.

In the HCP dataset, individual-specific MS-HBM parcellations also exhibited greater task functional homogeneity than parcellations from 4 other approaches (Supplementary Fig. S6), suggesting that MS-HBM parcellations better capture the "extrinsic" organization of individuals' cerebral cortex. Given the strong link between task-fMRI and rs-fMRI (Smith et al. 2009; Mennes et al. 2010; Cole et al. 2014; Krienen et al. 2014; Bertolero et al. 2015; Yeo, Krienen, et al. 2015a; Tavor et al. 2016; Gordon, Laumann, Gilmore, Newbold, et al. 2017c), this might not seem surprising. However, it is worth pointing out that the group-level parcellation performed as well as, if not better than 2 of the individual-specific parcellation approaches (Supplementary Fig. S6). Furthermore, the MS-HBM only demonstrated small improvements over the group-level parcellation in 5 of the 7 functional domains, while there was no statistical difference in the 2 remaining functional domains. One possibility is that the large-scale networks were too coarse to capture the finer details of task activation, consistent with our previous observation that the NeuroSynth forward inference maps do not cleanly match subtle features of the grouplevel large-scale networks. For example, the right-hand motor task preferentially activates the hand region of the left somatomotor cortex. However, Somatomotor A network covers the hand, foot and body regions of bilateral somatomotor cortex. As such, even if individual-specific Somatomotor A network were highly accurate, the resulting task inhomogeneity would still be relatively high.

\section{MS-HBM Approach Works Well With Single-Session rs-fMRI Data}

Increasing the scan duration of rs-fMRI can improve the reliability of functional connectivity measures (Van Dijk et al. 2010; $\mathrm{Xu}$ et al. 2016). While earlier studies have suggested that 5-12 min of resting-state scan might be sufficient to provide reliable measurements (Van Dijk et al. 2010; Birn et al. 2013), more recent studies have suggested the need for 25-30 min of data (Anderson et al. 2011; Laumann et al. 2015; Gordon, Laumann, Gilmore, Newbold, et al. 2017c). However, it is important to note that the amount of data necessary for reliable measurements depends on the functional connectivity measures being computed (Gordon, Laumann, Gilmore, Newbold, et al. 2017c), as well as the methods employed.

Consistent with previous studies, our experiments showed that the quality of individual-specific parcellations improved with more rs-fMRI data, although the improvements plateaued after around 30-40 min of data (Fig. 3B). Importantly, even though the MS-HBM was developed for multi-session rs-fMRI, the algorithm performed well even with single-session data. For example, the individual-specific MS-HBM parcellations estimated with one rs-fMRI session (10 min) exhibited comparable resting-state connectional homogeneity with parcellations estimated by 2 prominent approaches using 5 times the amount of data (Wang et al. 2015; Gordon, Laumann, Adeyemo, Petersen, et al. 2017a; Gordon, Laumann, Adeyemo, Gilmore, et al. 2017b). This improvement was made possible by exploiting prior information (e.g., group-level connectivity profiles, inter-subject RSFC variability and intra-subject RSFC variability) learned from multi-session rs-fMRI data. We expect high-quality individual-specific parcellations to require significantly longer scan time if no such prior information was used. 


\section{Methodological Considerations}

Consistent with recent studies (Mueller et al. 2013; Chen et al. 2015; Laumann et al. 2015), we found that association networks exhibited higher inter-subject RSFC variability than sensorymotor networks (Fig. 2 and Supplementary Figs S1 and S2). One important methodological consideration is that previous studies assumed functional correspondence across subjects after macro-anatomical alignment (Mueller et al. 2013; Chen et al. 2015; Laumann et al. 2015). However, it is well known that macro-anatomical alignment (or even functional alignment) is not sufficient to achieve perfect functional correspondence across subjects (Fischl, Sereno, Tootell, et al. 1999b; Yeo, Krienen, et al. 2010a; Harrison et al. 2015; Glasser et al. 2016; Langs et al. 2016). Indeed, a portion of the inter-subject functional connectivity variability observed in previous studies might be the result of residual functional misalignment across subjects (Harrison et al. 2015; Bijsterbosch et al. 2018; Salehi et al. 2018).

Consequently, despite the use of functionally aligned HCP data, we avoided assuming vertex-level functional correspondence provided by the MSMAll functional alignment (Robinson et al. 2014). Instead, the MS-HBM estimated inter-subject and intra-subject variabilities at the network level, and also explicitly differentiated between inter-subject network spatial variability and inter-subject RSFC variability. This allowed the possibility that for certain networks, inter-subject variability might be attributed to spatial variability, rather than RSFC variability. Nevertheless, we found that networks with higher inter-subject functional connectivity variability (Fig. 2 and Supplementary Figs S1 and S2) also exhibited greater inter-subject spatial variability (Supplementary Figs S3-S5).

Although the MS-HBM approach did not account for intersite variability, we demonstrated that model parameters estimated from one site can generalize to another site with a different acquisition protocol and scanner (Fig. 3, Supplementary Figs S8, S9, and S11). Given the increasing availability of multi-session rsfMRI from many different research groups (Zuo et al. 2014; Holmes et al. 2015; Poldrack et al. 2015; Filevich et al. 2017; Gordon, Laumann, Gilmore, Newbold, et al. 2017c), it might be possible to add another layer to the hierarchical model to account for intersite variability, in addition to intra-subject and inter-subject variabilities. Furthermore, our experiments did not differentiate between rs-fMRI runs collected within the same session versus rs-fMRI runs collected from different sessions. Another layer could again be inserted into the model to differentiate between within-subject intrasession and within-subject intersession variability. However, we suspect diminishing returns.

\section{Conclusions}

We developed a MS-HBM that differentiated between inter-subject and intra-subject variabilities when estimating individualspecific cortical network parcellations. Using a single rs-fMRI session (10 $\mathrm{min}$ ), our approach yielded parcellations comparable to those estimated by 2 state-of-the-art algorithms using 5 rs-fMRI sessions ( $50 \mathrm{~min}$ ), as evaluated by generalizability to new rs-fMRI data from the same subjects. Furthermore, intersubject variation in the spatial topography (location and arrangement) of cortical networks could be used to predict inter-subject variation in behavior, suggesting their potential utility as fingerprints of human behavior. Finally, spatial topography estimated by MS-HBM was more effective for behavioral prediction than spatial topography estimated by alternative parcellation approaches.

\section{Supplementary Material}

Supplementary material is available at Cerebral Cortex online.

\section{Funding}

This work was supported by Singapore Ministry of Education Tier 2 (MOE2014-T2-2-016), National University of Singapore (NUS) Strategic Research (DPRT/944/09/14), NUS School of Medicine Aspiration Fund (R185000271720), Singapore National Medical Research Council (CBRG/0088/2015), NUS Young Investigator Award, and the Singapore National Research Foundation (NRF) Fellowship (Class of 2017). Computational work for this article was partially performed on resources of the National Supercomputing Centre, Singapore (https://www.nscc. sg). H.L. was supported by the National Institute of Mental Health (1R01NS091604, P50MH106435) and the Beijing Municipal Science \& Technology Commission (Z161100002616009). A.J.H. was supported by the National Institute of Mental Health (573 K01MH099232). X.N.Z. was supported by the National Basic Research (973) Program (2015CB351702), the Natural Science Foundation of China (81471740, 81220108014), the National R\&D Infrastructure and Facility Development Program of China (DKA2017-12-02-21), and the Beijing Municipal Science and Tech Commission (Z161100002616023, Z171100000117012). S.B.E. was supported by the Deutsche Forschungsgemeinschaft (DFG, EI 816/11-1), the National Institute of Mental Health (R01MH074457), the Helmholtz Portfolio Theme "Supercomputing and Modeling for the Human Brain", and the European Union's Horizon 2020 Research and Innovation Programme under Grant Agreement No. 7202070 (HBP SGA1). Our research also utilized resources provided by the Center for Functional Neuroimaging Technologies, P41EB015896 and instruments supported by 1S10RR023401, 1S10RR019307, and 1S10RR023043 from the Athinoula A. Martinos Center for Biomedical Imaging at the Massachusetts General Hospital. Data were provided by the Brain Genomics Superstruct Project of Harvard University and the Massachusetts General Hospital (Principal Investigators: Randy Buckner, Joshua Roffman, and Jordan Smoller), with support from the Center for Brain Science Neuroinformatics Research Group, the Athinoula A. Martinos Center for Biomedical Imaging, and the Center for Human Genetic Research. Twenty individual investigators at Harvard and MGH generously contributed data to the overall project. Data were also provided by the Human Connectome Project, WU-Minn Consortium (Principal Investigators: David Van Essen and Kamil Ugurbil; 1U54MH091657) funded by the $16 \mathrm{NIH}$ Institutes and Centers that support the $\mathrm{NIH}$ Blueprint for Neuroscience Research; and by the McDonnell Center for Systems Neuroscience at Washington University.

\section{Notes}

We like to thank Julien Dubois for providing us with the corrected NEO5 personality scores for the HCP dataset. Conflict of Interest: None declared

\section{References}

Amunts K, Zilles K. 2015. Architectonic mapping of the human brain beyond Brodmann. Neuron. 88:1086-1107.

Anderson JS, Ferguson MA, Lopez-Larson M, Yurgelun-Todd D. 2011. Reproducibility of single-subject functional connectivity measurements. AJNR Am J Neuroradiol. 32:548-555.

Beckmann CF, DeLuca M, Devlin JT, Smith SM. 2005. Investigations into resting-state connectivity using 
independent component analysis. Philos Trans R Soc B Biol Sci. 360:1001-1013.

Beckmann C, Mackay C, Filippini N, Smith S 2009. Group comparison of resting-state fMRI data using multi-subject ICA and dual regression. 15th Annual Meeting of Organization for Human Brain Mapping. poster 441 SU-AM.

Bellec P, Rosa-Neto P, Lyttelton OC, Benali H, Evans AC. 2010. Multi-level bootstrap analysis of stable clusters in restingstate fMRI. Neuroimage. 51:1126-1139.

Bertolero MA, Yeo BTT, D’Esposito M. 2015. The modular and integrative functional architecture of the human brain. Proc Natl Acad Sci. 112:E6798-E6807.

Bijsterbosch JD, Woolrich MW, Glasser MF, Robinson EC, Beckmann CF, Van Essen DC, Harrison SJ, Smith SM. 2018. The relationship between spatial configuration and functional connectivity of brain regions. eLife. 7:e32992.

Birn RM, Molloy EK, Patriat R, Parker T, Meier TB, Kirk GR, Nair VA, Meyerand ME, Prabhakaran V. 2013. The effect of scan length on the reliability of resting-state fMRI connectivity estimates. Neuroimage. 83:550-558.

Biswal B, Zerrin Yetkin F, Haughton VM, Hyde JS. 1995. Functional connectivity in the motor cortex of resting human brain using echo-planar MRI. Magn Reson Med. 34: 537-541.

Braga RM, Buckner RL. 2017. Parallel interdigitated distributed networks within the individual estimated by intrinsic functional connectivity. Neuron. 95:457-471.

Buckner RL, Krienen FM, Castellanos A, Diaz JC, Yeo BTT. 2011. The organization of the human cerebellum estimated by intrinsic functional connectivity. J Neurophysiol. 106: 2322-2345.

Buckner RL, Krienen FM, Yeo BTT. 2013. Opportunities and limitations of intrinsic functional connectivity MRI. Nat Neurosci. 16:832-837.

Burgess GC, Kandala S, Nolan D, Laumann TO, Power JD, Adeyemo B, Harms MP, Petersen SE, Barch DM. 2016. Evaluation of denoising strategies to address motioncorrelated artifacts in resting-state functional magnetic resonance imaging data from the Human Connectome Project. Brain Connect. 6:669-680.

Cachia A, Roell M, Mangin J-F, Sun ZY, Jobert A, Braga L, Houde O, Dehaene S, Borst G. 2017. How interindividual differences in brain anatomy shape reading accuracy. Brain Struct Funct. 223:701-712.

Calhoun VD, Adali T. 2012. Multisubject independent component analysis of fMRI: a decade of intrinsic networks, default mode, and neurodiagnostic discovery. IEEE Rev Biomed Eng. 5:60-73.

Calhoun VD, Liu J, Adalı T. 2009. A review of group ICA for fMRI data and ICA for joint inference of imaging, genetic, and ERP data. Neuroimage. 45:S163-S172.

Chen B, Xu T, Zhou C, Wang L, Yang N, Wang Z, Dong H-M, Yang Z, Zang Y-F, Zuo X-N, et al. 2015. Individual variability and test-retest reliability revealed by ten repeated restingstate brain scans over one month. PLoS One. 10:e0144963.

Chong M, Bhushan C, Joshi AA, Choi S, Haldar JP, Shattuck DW, Spreng RN, Leahy RM. 2017. Individual parcellation of resting fMRI with a group functional connectivity prior. Neuroimage. 156:87-100.

Churchland PS, Sejnowski TJ. 1988. Perspectives on cognitive neuroscience. Science. 242:741-745.

Cole MW, Bassett DS, Power JD, Braver TS, Petersen SE. 2014. Intrinsic and task-evoked network architectures of the human brain. Neuron. 83:238-251.
Dale AM, Fischl B, Sereno MI. 1999. Cortical surface-based analysis. I. Segmentation and surface reconstruction. Neuroimage. 9:179-194.

Damoiseaux JS, Rombouts SARB, Barkhof F, Scheltens P, Stam CJ, Smith SM, Beckmann CF. 2006. Consistent resting-state networks across healthy subjects. Proc Natl Acad Sci USA. 103:13848-13853.

Dehaene S, Pegado F, Braga LW, Ventura P, Filho GN, Jobert A, Dehaene-Lambertz G, Kolinsky R, Morais J, Cohen L. 2010. How learning to read changes the cortical networks for vision and language. Science. 330:1359-1364.

Dosenbach NUF, Fair DA, Miezin FM, Cohen AL, Wenger KK, Dosenbach RAT, Fox MD, Snyder AZ, Vincent JL, Raichle ME, et al. 2007. Distinct brain networks for adaptive and stable task control in humans. Proc Natl Acad Sci. 104:11073-11078.

Dubois J, Galdi P, Han Y, Paul LK, Adolphs R. 2017. Resting-state functional brain connectivity best predicts the personality dimension of openness to experience. doi:https://doi.org/10. 1101/215129.

Eickhoff SB, Constable RT, Yeo BTT. 2018. Topographic organization of the cerebral cortex and brain cartography. Neuroimage. 170:332-347.

Erickson KI, Voss MW, Prakash RS, Basak C, Szabo A, Chaddock L, Kim JS, Heo S, Alves H, White SM, et al. 2011. Exercise training increases size of hippocampus and improves memory. Proc Natl Acad Sci. 108:3017-3022.

Felleman DJ, Van Essen DC. 1991. Distributed hierarchical processing in the primate cerebral cortex. Cereb Cortex. 1:1-47.

Filevich E, Lisofsky $\mathrm{N}$, Becker $\mathrm{M}$, Butler O, Lochstet $\mathrm{M}$, Martensson J, Wenger E, Lindenberger U, Kühn S. 2017. Day2day: investigating daily variability of magnetic resonance imaging measures over half a year. BMC Neurosci. 18: 161.

Filippini N, MacIntosh BJ, Hough MG, Goodwin GM, Frisoni GB, Smith SM, Matthews PM, Beckmann CF, Mackay CE. 2009. Distinct patterns of brain activity in young carriers of the APOE- $\varepsilon 4$ allele. Proc Natl Acad Sci. 106:7209-7214.

Finn ES, Shen X, Scheinost D, Rosenberg MD, Huang J, Chun MM, Papademetris X, Constable RT. 2015. Functional connectome fingerprinting: identifying individuals using patterns of brain connectivity. Nat Neurosci. 18:1664-1671.

Fischl B, Liu A, Dale AM. 2001. Automated manifold surgery: constructing geometrically accurate and topologically correct models of the human cerebral cortex. IEEE Trans Med Imaging. 20:70-80.

Fischl B, Sereno MI, Dale AM. 1999a. Cortical surface-based analysis. II: inflation, flattening, and a surface-based coordinate system. Neuroimage. 9:195-207.

Fischl B, Sereno MI, Tootell RBH, Dale AM. 1999b. Highresolution inter-subject averaging and a coordinate system for the cortical surface. Hum Brain Mapp. 8:272-284.

Fischl B, Van Der Kouwe A, Destrieux C, Halgren E, Ségonne F, Salat DH, Busa E, Seidman LJ, Goldstein J, Kennedy D, et al. 2004. Automatically parcellating the human cerebral cortex. Cereb Cortex. 14:11-22.

Fox MD, Corbetta M, Snyder AZ, Vincent JL, Raichle ME. 2006. Spontaneous neuronal activity distinguishes human dorsal and ventral attention systems. Proc Natl Acad Sci. 103: 10046-10051.

Fox MD, Raichle ME. 2007. Spontaneous fluctuations in brain activity observed with functional magnetic resonance imaging. Nat Rev Neurosci. 8:700-711.

Ge T, Holmes AJ, Buckner RL, Smoller JW, Sabuncu MR. 2017. Heritability analysis with repeat measurements and its 
application to resting-state functional connectivity. Proc Natl Acad Sci. 114:5521-5526.

Glahn DC, Winkler AM, Kochunov P, Almasy L, Duggirala R, Carless MA, Curran JC, Olvera RL, Laird AR, Smith SM, et al. 2010. Genetic control over the resting brain. Proc Natl Acad Sci USA. 107:1223-1228.

Glasser MF, Coalson TS, Robinson EC, Hacker CD, Harwell J, Yacoub E, Uğurbil K, Andersson J, Beckmann CF, Jenkinson $\mathrm{M}$, et al. 2016. A multi-modal parcellation of human cerebral cortex. Nature. 536:171-178.

Glasser MF, Sotiropoulos SN, Wilson JA, Coalson T, Fischl B, Andersson JL, Xu J, Jbabdi S, Webster M, Polimeni JR, et al. 2013. The minimal preprocessing pipelines for the Human Connectome Project. Neuroimage. 80:105-124.

Gordon EM, Laumann TO, Adeyemo B, Gilmore AW, Nelson SM, Dosenbach NUF, Petersen SE. 2017b. Individual-specific features of brain systems identified with resting state functional correlations. Neuroimage. 146:918-939.

Gordon EM, Laumann TO, Adeyemo B, Huckins JF, Kelley WM, Petersen SE. 2016. Generation and evaluation of a cortical area parcellation from resting-state correlations. Cereb Cortex. 26:288-303.

Gordon EM, Laumann TO, Adeyemo B, Petersen SE. 2017a. Individual variability of the system-level organization of the human brain. Cereb Cortex. 27:386-399.

Gordon EM, Laumann TO, Gilmore AW, Newbold DJ, Greene DJ, Berg JJ, Ortega M, Hoyt-Drazen C, Gratton C, Sun H, et al. 2017c. Precision functional mapping of individual human brains. Neuron. 95:791-807.e7.

Greicius MD, Krasnow B, Reiss AL, Menon V. 2003. Functional connectivity in the resting brain: a network analysis of the default mode hypothesis. Proc Natl Acad Sci. 100: 253-258.

Greve DN, Fischl B. 2009. Accurate and robust brain image alignment using boundary-based registration. Neuroimage. 48:63-72.

Griffanti L, Salimi-Khorshidi G, Beckmann CF, Auerbach EJ, Douaud G, Sexton CE, Zsoldos E, Ebmeier KP, Filippini N, Mackay CE, et al. 2014. ICA-based artefact removal and accelerated fMRI acquisition for improved resting state network imaging. Neuroimage. 95:232-247.

Hacker CD, Laumann TO, Szrama NP, Baldassarre A, Snyder AZ, Leuthardt EC, Corbetta M. 2013. Resting state network estimation in individual subjects. Neuroimage. 82:616-633.

Hampson M, Driesen NR, Skudlarski P, Gore JC, Constable RT. 2006. Brain connectivity related to working memory performance. J Neurosci. 26:13338-13343.

Harrison SJ, Woolrich MW, Robinson EC, Glasser MF, Beckmann CF, Jenkinson M, Smith SM. 2015. Large-scale probabilistic functional modes from resting state fMRI. Neuroimage. 109: 217-231.

Hawrylycz M, Miller JA, Menon V, Feng D, Dolbeare T, GuillozetBongaarts AL, Jegga AG, Aronow BJ, Lee C-K, Bernard A, et al. 2015. Canonical genetic signatures of the adult human brain. Nat Neurosci. 18:1832-1844.

Holmes AJ, Hollinshead MO, O'Keefe TM, Petrov VI, Fariello GR, Wald LL, Fischl B, Rosen BR, Mair RW, Roffman JL, et al. 2015. Brain Genomics Superstruct Project initial data release with structural, functional, and behavioral measures. Sci data. 2:150031.

Holmes AJ, Hollinshead MO, Roffman JL, Smoller JW, Buckner RL. 2016. Individual differences in cognitive control circuit anatomy link sensation seeking, impulsivity, and substance use. J Neurosci. 36:4038-4049.
Jenkinson M, Bannister P, Brady M, Smith S. 2002. Improved optimization for the robust and accurate linear registration and motion correction of brain images. Neuroimage. 17:825-841.

Kaas JH. 1987. The organization of neocortex in mammals: implications for theories of brain function. Annu Rev Psychol. 38:129-151.

Krienen FM, Yeo BTT, Buckner RL. 2014. Reconfigurable taskdependent functional coupling modes cluster around a core functional architecture. Phil Trans R Soc B. 369:20130526.

Krienen FM, Yeo BTT, Ge T, Buckner RL, Sherwood CC. 2016. Transcriptional profiles of supragranular-enriched genes associate with corticocortical network architecture in the human brain. Proc Natl Acad Sci. 113:E469-E478.

Langs G, Wang D, Golland P, Mueller S, Pan R, Sabuncu MR, Sun W, Li K, Liu H. 2016. Identifying shared brain networks in individuals by decoupling functional and anatomical variability. Cereb Cortex. 26:4004-4014.

Lashkari D, Vul E, Kanwisher N, Golland P. 2010. Discovering structure in the space of fMRI selectivity profiles. Neuroimage. 50:1085-1098.

Laumann TO, Gordon EM, Adeyemo B, Snyder AZ, Joo SJ, Chen MY, Gilmore AW, McDermott KB, Nelson SM, Dosenbach NUF, et al. 2015. Functional system and areal organization of a highly sampled individual human brain. Neuron. 87:657-670.

Lee MH, Hacker CD, Snyder AZ, Corbetta M, Zhang D, Leuthardt EC, Shimony JS. 2012. Clustering of resting state networks. PLoS One. 7:e40370.

Lindemer ER, Greve DN, Fischl BR, Augustinack JC, Salat DH. 2017. Regional staging of white matter signal abnormalities in aging and Alzheimer's disease. Neuroimage: Clin. 14: 156-165.

Margulies DS, Kelly AMC, Uddin LQ Biswal BB, Castellanos FX, Milham MP. 2007. Mapping the functional connectivity of anterior cingulate cortex. Neuroimage. 37:579-588.

Mennes M, Kelly C, Zuo X-N, Di Martino A, Biswal BB, Castellanos FX, Milham MP. 2010. Inter-individual differences in resting-state functional connectivity predict taskinduced BOLD activity. Neuroimage. 50:1690-1701.

Misra C, Fan Y, Davatzikos C. 2009. Baseline and longitudinal patterns of brain atrophy in $\mathrm{MCI}$ patients, and their use in prediction of short-term conversion to $\mathrm{AD}$ : results from ADNI. Neuroimage. 44:1415-1422.

Mueller S, Wang D, Fox MD, Yeo BTT, Sepulcre J, Sabuncu MR, Shafee R, Lu J, Liu H. 2013. Individual variability in functional connectivity architecture of the human brain. Neuron. 77:586-595.

Murphy KP. 2012. Machine learning: a probabilistic perspective. Cambridge (MA): MIT Press.

Noble S, Spann MN, Tokoglu F, Shen X, Constable RT, Scheinost D. 2017. Influences on the test-retest reliability of functional connectivity MRI and its relationship with behavioral utility. Cereb Cortex. 27:5415-5429.

Nostro AD, Müller VI, Varikuti DP, Pläschke RN, Hoffstaedter F, Langner R, Patil KR, Eickhoff SB. 2018. Predicting personality from network-based resting-state functional connectivity. Brain Struct Funct. 10.1007/s00429-018-1651-z.

Poldrack RA, Laumann TO, Koyejo O, Gregory B, Hover A, Chen M-Y, Gorgolewski KJ, Luci J, Joo SJ, Boyd RL, et al. 2015. Longterm neural and physiological phenotyping of a single human. Nat Commun. 6:8885.

Power JD, Cohen AL, Nelson SM, Wig GS, Barnes KA, Church JA, Vogel AC, Laumann TO, Miezin FM, Schlaggar BL, et al. 2011. Functional network organization of the human brain. Neuron. 72:665-678. 
Power JD, Mitra A, Laumann TO, Snyder AZ, Schlaggar BL, Petersen SE. 2014. Methods to detect, characterize, and remove motion artifact in resting state fMRI. Neuroimage. 84:320-341.

Raj A, LoCastro E, Kuceyeski A, Tosun D, Relkin N, Weiner M. 2015. Network diffusion model of progression predicts longitudinal patterns of atrophy and metabolism in Alzheimer's disease. Cell Rep. 10:359-369.

Richiardi J, Altmann A, Milazzo A-C, Chang C, Chakravarty MM, Banaschewski T, Barker GJ, Bokde ALW, Bromberg U, Büchel C, et al. 2015. Correlated gene expression supports synchronous activity in brain networks. Science. 348:1241-1244.

Risacher SL, Shen L, West JD, Kim S, McDonald BC, Beckett LA, Harvey DJ, Jack CR Jr., Weiner MW, Saykin AJ. 2010. Longitudinal MRI atrophy biomarkers: relationship to conversion in the ADNI cohort. Neurobiol Aging. 31:1401-1418.

Robinson EC, Jbabdi S, Glasser MF, Andersson J, Burgess GC, Harms MP, Smith SM, Van Essen DC, Jenkinson M. 2014. MSM: a new flexible framework for multimodal surface matching. Neuroimage. 100:414-426.

Rosenberg MD, Finn ES, Scheinost D, Papademetris X, Shen X, Constable RT, Chun MM. 2016. A neuromarker of sustained attention from whole-brain functional connectivity. Nat Neurosci. 19:165-171.

Sabuncu MR, Ge T, Holmes AJ, Smoller JW, Buckner RL, Fischl B, the Alzheimer's Disease Neuroimaging Initiative. 2016. Morphometricity as a measure of the neuroanatomical signature of a trait. Proc Natl Acad Sci. 113:E5749-E5756.

Salehi M, Karbasi A, Shen X, Scheinost D, Constable RT. 2018. An exemplar-based approach to individualized parcellation reveals the need for sex specific functional networks. 170: 54-67.

Salimi-Khorshidi G, Douaud G, Beckmann CF, Glasser MF, Griffanti L, Smith SM. 2014. Automatic denoising of functional MRI Data: Combining independent component analysis and hierarchical fusion of classifiers. Neuroimage. 90: 449-468.

Schaefer A, Kong R, Gordon EM, Laumann TO, Zuo X-N, Holmes AJ, Eickhoff SB, Yeo BTT. 2017. Local-global parcellation of the human cerebral cortex from intrinsic functional connectivity MRI. Cereb Cortex. In press. doi:10.1093/cercor/bhx179

Seeley WW, Menon V, Schatzberg AF, Keller J, Glover GH, Kenna H, Reiss AL, Greicius MD. 2007. Dissociable intrinsic connectivity networks for salience processing and executive control. J Neurosci. 27:2349-2356.

Ségonne F, Pacheco J, Fischl B. 2007. Geometrically accurate topology-correction of cortical surfaces using nonseparating loops. IEEE Trans Med Imaging. 26:518-529.

Shine JM, Koyejo O, Poldrack RA. 2016. Temporal metastates are associated with differential patterns of time-resolved connectivity, network topology, and attention. Proc Natl Acad Sci. 113:9888-9891.

Siegel JS, Mitra A, Laumann TO, Seitzman BA, Raichle M, Corbetta M, Snyder AZ. 2017. Data quality influences observed links between functional connectivity and behavior. Cereb Cortex. 27:4492-4502.

Smith SM, Beckmann CF, Andersson J, Auerbach EJ, Bijsterbosch J, Douaud G, Duff E, Feinberg DA, Griffanti L, Harms MP, et al. 2013. Resting-state fMRI in the Human Connectome Project. Neuroimage. 80:144-168.

Smith SM, Fox PT, Miller KL, Glahn DC, Fox PM, Mackay CE, Filippini N, Watkins KE, Toro R, Laird AR, et al. 2009. Correspondence of the brain's functional architecture during activation and rest. Proc Natl Acad Sci. 106:13040-13045.
Smith SM, Jenkinson M, Woolrich MW, Beckmann CF, Behrens TEJ, Johansen-Berg H, Bannister PR, De Luca M, Drobnjak I, Flitney D, et al. 2004. Advances in functional and structural MR image analysis and implementation as FSL. Neuroimage. 23:S208-S219.

Smith SM, Nichols TE, Vidaurre D, Winkler AM, Behrens TE, Glasser MF, Ugurbil K, Barch DM, Van Essen DC, Miller KL. 2015. A positive-negative mode of population covariation links brain connectivity, demographics and behavior. Nat Neurosci. 18:1565-1567.

Tagliazucchi E, Laufs H. 2014. Decoding wakefulness levels from typical fMRI resting-state data reveals reliable drifts between wakefulness and sleep. Neuron. 82:695-708.

Tavor I, Jones OP, Mars RB, Smith SM, Behrens TE, Jbabdi S. 2016. Task-free MRI predicts individual differences in brain activity during task performance. Science. 352:216-220.

van den Heuvel MP, Stam CJ, Kahn RS, Hulshoff Pol HE. 2009. Efficiency of functional brain networks and intellectual performance. J Neurosci. 29:7619-7624.

Van Dijk KRA, Hedden T, Venkataraman A, Evans KC, Lazar SW, Buckner RL. 2010. Intrinsic functional connectivity as a tool for human connectomics: theory, properties, and optimization. J Neurophysiol. 103:297-321.

Van Essen DC, Glasser MF, Dierker DL, Harwell J, Coalson T. 2012a. Parcellations and hemispheric asymmetries of human cerebral cortex analyzed on surface-based atlases. Cereb Cortex. 22:2241-2262.

Van Essen DC, Ugurbil K, Auerbach E, Barch D, Behrens TEJ, Bucholz R, Chang A, Chen L, Corbetta M, Curtiss SW, et al. 2012b. The Human Connectome Project: a data acquisition perspective. Neuroimage. 62:2222-2231.

Varoquaux G, Gramfort A, Pedregosa F, Michel V, Thirion B. 2011. Multi-subject dictionary learning to segment an atlas of brain spontaneous activity. Inf Process Med Imaging. 22:562-573.

Varoquaux G, Raamana PR, Engemann DA, Hoyos-Idrobo A, Schwartz Y, Thirion B. 2017. Assessing and tuning brain decoders: cross-validation, caveats, and guidelines. Neuroimage. 145:166-179.

Wang D, Buckner RL, Fox MD, Holt DJ, Holmes AJ, Stoecklein S, Langs G, Pan R, Qian T, Li K, et al. 2015. Parcellating cortical functional networks in individuals. Nat Neurosci. 18:1853-1860.

Wang C, Ong JL, Patanaik A, Zhou J, Chee MWL. 2016. Spontaneous eyelid closures link vigilance fluctuation with fMRI dynamic connectivity states. Proc Natl Acad Sci. 113: 9653-9658.

Wig GS. 2017. Segregated systems of human brain networks. Trends Cogn Sci. 21:981-996.

Wig GS, Laumann TO, Cohen AL, Power JD, Nelson SM, Glasser MF, Miezin FM, Snyder AZ, Schlaggar BL, Petersen SE. 2014. Parcellating an individual subject's cortical and subcortical brain structures using snowball sampling of resting-state correlations. Cereb Cortex. 24:2036-2054.

Xu T, Opitz A, Craddock RC, Wright MJ, Zuo X-N, Milham MP. 2016. Assessing variations in areal organization for the intrinsic brain: from fingerprints to reliability. Cereb Cortex. 26:4192-4211.

Yang Z, Zuo X-N, McMahon KL, Craddock RC, Kelly C, de Zubicaray GI, Hickie I, Bandettini PA, Castellanos FX, Milham MP, et al. 2016. Genetic and environmental contributions to functional connectivity architecture of the human brain. Cereb Cortex. 26:2341-2352.

Yarkoni T, Poldrack RA, Nichols TE, Van Essen DC, Wager TD. 2011. Large-scale automated synthesis of human functional neuroimaging data. Nat Methods. 8:665-670. 
Yeo BTT, Krienen FM, Eickhoff SB, Yaakub SN, Fox PT, Buckner RL, Asplund CL, Chee MWL. 2015a. Functional specialization and flexibility in human association cortex. Cereb Cortex. 25:3654-3672.

Yeo BTT, Krienen FM, Sepulcre J, Sabuncu MR, Lashkari D, Hollinshead M, Roffman JL, Smoller JW, Zöllei L, Polimeni JR, et al. 2011. The organization of the human cerebral cortex estimated by intrinsic functional connectivity. J Neurophysiol. 106: 1125-1165.

Yeo BTT, Sabuncu MR, Vercauteren T, Ayache N, Fischl B, Golland P. 2010a. Spherical Demons: fast diffeomorphic landmark-free surface registration. IEEE Trans Med Imaging. 29:650-668.

Yeo BTT, Tandi J, Chee MWL. 2015b. Functional connectivity during rested wakefulness predicts vulnerability to sleep deprivation. Neuroimage. 111:147-158.
Zeng LL, Wang D, Fox MD, Sabuncu M, Hu D, Ge M, Buckner RL, Liu H. 2014. Neurobiological basis of head motion in brain imaging. Proc Natl Acad Sci. 111:6058-6062.

Zhang X, Mormino EC, Sun N, Sperling RA, Sabuncu MR, Yeo BTT, the Alzheimer's Disease Neuroimaging Initiative. 2016. Bayesian model reveals latent atrophy factors with dissociable cognitive trajectories in Alzheimer's disease. Proc Natl Acad Sci. 113:E6535-E6544.

Zuo XN, Anderson JS, Bellec P, Birn RM, Biswal BB, Blautzik J, Breitner JC, Buckner RL, Calhoun VD, Castellanos FX, et al. 2014. An open science resource for establishing reliability and reproducibility in functional connectomics. Sci Data. 1:140049.

Zuo XN, Kelly C, Adelstein JS, Klein DF, Castellanos FX, Milham MP. 2010. Reliable intrinsic connectivity networks: Testretest evaluation using ICA and dual regression approach. Neuroimage. 49:2163-2177. 